\title{
Article \\ Adjunctive Effect of Systemic Antibiotics in Regenerative/Reconstructive Periodontal Surgery- A Systematic Review with Meta-Analysis
}

\author{
Luigi Nibali ${ }^{1, *}$, Jacopo Buti ${ }^{2}$, Luigi Barbato ${ }^{3}$, Francesco Cairo ${ }^{3}$, Filippo Graziani ${ }^{4}$ and Søren Jepsen ${ }^{5}$ \\ 1 Periodontology Unit, Centre for Host Microbiome Interactions, King's College London, London SE1 9RT, UK \\ 2 Periodontology Department, UCL Eastman Dental Institute, University College London, \\ London WC1E 6BT, UK; j.buti@ucl.ac.uk \\ 3 Research Unit in Periodontology and Periodontal Medicine, Department of Experimental and Clinical \\ Medicine, University of Florence, 50121 Florence, Italy; luigi.barbato85@libero.it (L.B.); \\ cairofrancesco@virgilio.it (F.C.) \\ 4 Unit of Dentistry and Oral Surgery, Department of Surgical, Medical and Molecular Pathology and \\ CriticalCare Medicine, University of Pisa, 56126 Pisa, Italy; filippo.graziani@med.unipi.it \\ 5 Department of Periodontology, Operative \& Preventive Dentistry, University Hospital Bonn, \\ 53127 Bonn, Germany; sjepsen@uni-bonn.de \\ * Correspondence: luigi.nibali@kcl.ac.uk
}

check for updates

Citation: Nibali, L.; Buti, J.; Barbato, L.; Cairo, F.; Graziani, F.; Jepsen, S. Adjunctive Effect of Systemic Antibiotics in Regenerative/ Reconstructive Periodontal SurgeryA Systematic Review with MetaAnalysis. Antibiotics 2022, 11, 8. https://doi.org/10.3390/antibiotics 11010008

Academic Editors: Andreas Braun and Felix Krause

Received: 30 October 2021

Accepted: 13 December 2021

Published: 22 December 2021

Publisher's Note: MDPI stays neutral with regard to jurisdictional claims in published maps and institutional affiliations.

Copyright: (C) 2021 by the authors. Licensee MDPI, Basel, Switzerland. This article is an open access article distributed under the terms and conditions of the Creative Commons Attribution (CC BY) license (https:// creativecommons.org/licenses/by/ $4.0 /)$.

\begin{abstract}
Background and Objective: Systemic antibiotics $(\mathrm{AB})$ are often used in conjunction with regenerative/reconstructive periodontal surgery of intrabony defects and furcations; however, their potential benefits have not been systematically assessed. Materials and Methods: Data were retrieved from two recent systematic reviews (a total of 105 randomized clinical trials (RCTs) on clinical and radiographic outcomes in intrabony defects (ID) and molars with furcation involvement (FI) treated by surgical access with regenerative techniques. Pair-wise meta-analysis of RCTs with and without $\mathrm{AB}$ was performed. Meta-regressions from single-arm (subgroup) RCTs including study arms with or without adjunctive $A B$ were also conducted. Results: No statistically significant benefits of systemic $\mathrm{AB}$ with regard to PPD, CAL and bone gain were detected in ID by pair-wise meta-analysis. Metaregression revealed increased PPD reduction $(-0.91 \mathrm{~mm}, 95 \% \mathrm{CI}=-1.30 ;-0.51, p<0.001)$, CAL gain $(-0.92 \mathrm{~mm}, 95 \% \mathrm{CI}=-1.32 ;-0.52, p<0.001)$ and bone gain $(-1.08 \mathrm{~mm}, 95 \% \mathrm{CI}=-1.63 ;-0.53$, $p<0.001)$ in ID but not in any of the outcomes in FI for arms treated with AB vs. study arms treated with no $A B$. No clear differences in adverse events were detected between $A B$ and non- $A B$ groups. Conclusion: There is only weak indirect evidence that $\mathrm{AB}$ may provide additional benefits in terms of clinical improvements in the regenerative/reconstructive periodontal surgery of intrabony defects and no evidence for a benefit in furcations. Until new data are gained and in the context of antibiotic stewardship, it may be questionable to justify the adjunctive use of systemic antibiotics.
\end{abstract}

Keywords: periodontitis; regeneration; antibiotic; infection; surgery

\section{Introduction}

The use of systemic antimicrobials in periodontal therapy has been advocated for several decades, owing to the importance of microbes as triggers of the periodontal pathogenic process [1,2]. Several studies focused on the potential role of systemic antibiotics as adjuncts to mechanical non-surgical therapy, with the aim to improve pocket depth reduction and attachment level gain. A recent systematic review (SR) and meta-analysis concluded that the use of systemic antimicrobials as an adjunct to subgingival instrumentation results in statistically significant improvements in clinical outcomes. Adverse events, albeit usually not severe, are more frequent when antibiotics rather than placebo are used [3]. However, there is rising concern about the global emergence of antibiotic resistance $[4,5]$. and a recent S3-level clinical guideline called for a restricted use of adjunctive systemic antibiotics during step 2 of periodontal therapy [6]. 
The other common application of systemic antibiotics is during periodontal surgery, and particularly regenerative/reconstructive surgery. These procedures aim to stimulate the creation of new periodontal attachment with the use of various types of biomaterials, leading to a higher magnitude of clinical benefits in terms of pocket reduction and clinical attachment gain compared with flap surgery alone [7,8]. Antibiotics are prescribed with the objective to prevent post-operative infections (may reduce post-surgical complications) and to enhance clinical outcomes. However, this aspect has been rather under-researched compared with the use of antibiotics during the non-surgical phase [9-11]. Therefore, in the reality of clinical practice, antibiotics are often empirically prescribed in the case of regenerative periodontal surgery, with no clear evidence to support it $[12,13]$. However, to date, the potential benefits of systemic antibiotics as an adjunct to regenerative periodontal surgery have not been systematically appraised.

Therefore, we aimed to investigate the adjunctive benefit of systemic antimicrobials on clinical and radiographic outcomes in intrabony defects (ID) and molars with furcation involvement $(\mathrm{FI})$ treated with periodontal regenerative/reconstructive surgery.

\section{Materials and Methods}

The PRISMA statement [14] was followed both in the planning [15] and reporting [16] of the review (Supplementary Material S1). Both original reviews providing data for the present review [7,8] had been registered on PROSPERO (ID references CRD42019124022 and CRD42019124466, respectively).

\subsection{Focused Question}

"In patients with periodontitis with ID or FI treated with regenerative surgery, what is the adjunctive benefit of systemic antimicrobials on clinical and radiographic outcomes in intrabony defects (ID) and molars with furcation involvement (FI) treated with periodontal regenerative/reconstructive surgery?"

\subsection{Eligibility Criteria}

The following PICOS method was the following:

- $\quad$ Population: patients with periodontitis with ID or FI treated with regenerative surgery.

- Intervention: adjunctive use of systemic antibiotics.

- Comparison: no use of systemic antibiotics.

- Outcomes: clinical attachment level (CAL) gain, probing pocket depth (PPD) reduction, bone gain.

- $\quad$ Studies: RCTs testing a regenerative technique at least in one arm and including at least 10 patients/arm and at least 1-year follow-up.

For this SR, we considered any type of regenerative surgery [guided tissue regeneration (GTR), enamel matrix derivative (EMD), bone filler or substitutes (BRG), growth factors (GF)] in patients with periodontal intra-bony defects $\geq 3 \mathrm{~mm}$ [7] or periodontitisrelated furcation defects of any type (class II and III) [8]. Combination therapies were also considered.

\subsection{Information Sources, Search and Study Selection}

The searches from two recent SRs $[7,8]$ were used as a starting point for this SR and the last update was performed on the 20th of November 2020. Briefly, all the studies included in the two SRs were reconsidered according to the PICO criteria for this review. Additionally, the list of the full texts excluded was also checked for additional titles. Then the searches strategies used in the previous reviews were updated. The new titles were screened, and full texts were evaluated according to the PICO criteria for this review (for additional information, see Supplementary Material S2). 


\subsection{Study Characteristics and Data Items}

All papers included were screened for reporting the use of antimicrobials. When this was not clearly stated, senior authors of the respective papers were contacted to enquire whether antimicrobials were used or not as part of the study protocol.

The following outcomes from the included studies were registered:

- $\quad$ For IDs: CAL gain, PPD reduction and bone gain [7]

- For FIs: horizontal clinical attachment level (H-CAL) gain, vertical clinical attachment level (V-CAL), (PPD) reduction, horizontal bone level (HBL) gain [8]

- Information regarding Adverse Events (AE) were also considered.

\subsection{Risk of Bias Analysis}

The quality of the included studies was assessed using the Cochrane Collaboration's Tool for assessing the risk of bias for RCTs [17]. The assessments of the risk of bias previously reported in Nibali et al. [7] and Jepsen et al. [8] were updated by adding the new papers included in this SR.

\subsection{Summary Measures and Planned Method of Analysis}

Data were grouped with respect to (a) regenerative biomaterials used (e.g., EMD, GTR-R, GTR-NR, BRG)—studies included EMD, GTR with resorbable and non-resorbable membranes, with or without bone replacement grafts; (b) antibiotics $(A B+) /$ non-antibiotics $(\mathrm{AB}-)$ for each of the outcome measures and analyzed separately for IDs (CAL gain, PPD reduction and bone gain) and FIs (H-CAL gain, V-CAL gain, PPD reduction and H-BL gain). Standard pair-wise meta-analyses were conducted when studies directly comparing regenerative techniques with and without the adjunctive use of antibiotics were available. Random-effects models were used throughout. For continuous data, pooled outcomes were expressed as mean differences with their associated 95\% confidence intervals (CI). The analyses were conducted using the generic inverse variance statistical method where the means and standard deviations (SD) were entered for each study arm. Statistical heterogeneity was assessed by calculation of the $\mathrm{Q}$ statistic. Analyses were performed using Review Manager (RevMan) [Computer program] (Version 5.3. Copenhagen: The Nordic Cochrane Centre, The Cochrane Collaboration, 2014). Furthermore, after grouping data with respect to the use or not of antibiotics $(\mathrm{AB}+/ \mathrm{AB}-)$, meta-analyses were performed, including individual arms of RCTs. The degree of heterogeneity between studies was quantified utilizing the $\mathrm{I}^{2}$ statistical test and subgroup and meta-regression analyses were performed to determine the effect of the adjunctive use of antibiotics (AB+vs. $A B-$ ) and the type of antibiotics (penicillin vs. tetracycline) using specific software for metaanalysis (OpenMeta[Analyst]) (open-source software, Brown University of Public Health, Providence, RI, USA). The DerSimonian-Laird random-effects method was chosen. Results were presented as Forest plots with weighted mean values and $95 \%$ confidence intervals (CI). A $p$ value $<0.05$ was considered statistically significant.

\section{Results}

A total of 105 studies were included in this SR (see Supplementary Material S3 for a full list of included papers). Out of these 105 studies, 86 accounting for 150 treatment arms reported data on IDs, while 19 accounting for 35 treatment arms reported data on FIs (see flowchart diagram in Supplementary Material S4). Out of 105 included studies, in 75 the use of systemic antibiotics was reported after the surgery; in two studies, the use of systemic antibiotics was reported only the day of the surgery, while in 24 studies, systemic antibiotics were not used. Only four studies tested a systemic antibiotic vs. no antibiotic. The most used antibiotics were amoxicillin and doxycycline. Tables 1 and 2 report details of antibiotic use in all included papers. 
Table 1. Characteristics of the treatment arms from included studies treating intra-bony defects (ID).

\begin{tabular}{|c|c|c|c|c|c|c|c|}
\hline Author/Treatment & Country & $\begin{array}{c}\mathbf{N}^{\circ} \\
\text { Patients }\end{array}$ & $\begin{array}{c}\mathbf{N}^{\circ} \\
\text { Defects }\end{array}$ & Antibiotic Regimen & CAL Gain & $\begin{array}{c}\text { PPD } \\
\text { Reduction }\end{array}$ & Bone Gain \\
\hline \multicolumn{8}{|l|}{ EMD } \\
\hline Silvestri et al. (OFD + EMD) 2000 & Italy & 10 & 10 & Amoxicillin 875 + clavulanic 125 b.i.d. for 7 days & $4.5 \pm 1.6$ & $4.8 \pm 1.6$ & - \\
\hline Okuda et al. (OFD + EMD) 2000 & Japan & 16 & 18 & Cafaclor 750 mg q.d. 5 days & $1.72 \pm 1.07$ & $3 \pm 0.97$ & - \\
\hline Crea et al. (OFD + EMD) 2008 & Italy & 19 & 19 & Amoxicillin $500 \mathrm{mg}$ b.i.d. for 7 days & $2.4 \pm 1.1$ & $3.1 \pm 1.4$ & $2.7 \pm 1$ \\
\hline Iorio-Siciliano et al. (OFD + EMD) 2011 & Italy & 20 & 20 & No antibiotic & $2.4 \pm 2.2$ & $2.9 \pm 2.1$ & - \\
\hline Pontoriero et al. (OFD + EMD) 1999 & Sweden & 10 & 10 & $3 \mathrm{G}$ amoxicillin $1 \mathrm{~h}$ before surgery & $2.9 *$ & $4.4 *$ & - \\
\hline Sanz et al. (OFD + EMD) 2004 & Spain & 35 & 35 & No antibiotics & $3.1 \pm 1.8$ & $3.8 \pm 1.5$ & - \\
\hline Minabe et al. (OFD + EMD) 2002 & Japan & - & 22 & Cefaclor $750 \mathrm{mg}$ q.d. for 4 days & $2.6 \pm 1$ & $3.8 \pm 0.9$ & - \\
\hline Guida et al. (OFD + EMD) 2007 & Italy & 14 & 14 & Amoxicillin 875 + clavulanic 125 b.i.d. for 6 days & $4.6 \pm 1.3$ & $5.6 \pm 1.7$ & $4.3 \pm 2.4$ \\
\hline Yilmaz et al. (OFD + EMD) 2010 & Turkey & 20 & 20 & Amoxicillin $500 \mathrm{mg}$ t.i.d. for 7 days & $3.4 \pm 0.8$ & $4.6 \pm 0.4$ & $2.8 \pm 0.8$ \\
\hline Losada et al. (OFD + EMD) 2016 & Spain & 23 & 23 & $\begin{array}{l}\text { Amoxicillin } 750 \text { or Clindamycin } 300 \text { mg t.i.d. } \\
\text { for } 7 \text { days }\end{array}$ & $2.65 \pm 2.18$ & $3.3 \pm 1.89$ & $2.6 \pm 2.03$ \\
\hline Meyle et al. (OFD + EMD) 2011 & Germany & 35 & 35 & No antibiotics & $1.93 \pm 1.7$ & $2.9 \pm 1.8$ & $2.81 \pm 1.6$ \\
\hline Pietruska et al. (OFD + EMD) 2011 & Poland & 12 & 12 & Amoxicillin $500 \mathrm{mg}$ t.i.d. for 7 days & $\S$ & $\S$ & - \\
\hline Sipos et al. (OFD + EMD) 2005 & Netherlands & 11 & 12 & No antibiotics & $1.28 \pm 2.04$ & $2.86 \pm 0.75$ & $1.63 \pm 1.21$ \\
\hline Leknes et al. (OFD + EMD) 2009 & Norway & 13 & 13 & No antibiotics & $0.6 \pm 1$ & $2.5 \pm 1.9$ & - \\
\hline Al Machot et al. (OFD + EMD) 2014 & Germany & 19 & 19 & No antibiotics & $1.4 \pm 1.8$ & $2.6 \pm 1.8$ & $1.6 \pm 1.2$ \\
\hline Eickholz et al. (OFD + EMD) 2014a & Germany & 28 & 28 & Doxycycline $200 \mathrm{mg}$ q.d. for 7 days & $2.74 \pm 1.89$ & $3.69 \pm 2.23$ & - \\
\hline Eickholz et al. (OFD + EMD) 2014b & Germany & 29 & 29 & No antibiotics & $2.95 \pm 1.92$ & $3.4 \pm 1.73$ & - \\
\hline De Leonardis et al. (PPF + EMD) 2013 & Italy & 34 & 34 & Amoxicillin $2 \mathrm{~g}$ q.d for 6 days & $2.95 \pm 0.74$ & $3.76 \pm 0.74$ & $2.61 \pm 0.49$ \\
\hline Fickl et al. (PPF + EMD) 2009 & Germany & 19 & 35 & No antibiotics & $3.7 \pm 0.4$ & $4.2 \pm 0.3$ & $2.5 \pm 0.4$ \\
\hline Francetti et al. (PPF + EMD) 2004 & Italy & 11 & 11 & $\begin{array}{l}\text { Amoxicillin } 875 \mathrm{mg}+\text { clavulanic } 125 \mathrm{mg} \text { b.i.d. } \\
\text { for } 5 \text { days }\end{array}$ & $4.29 \pm 1.38$ & $4.86 \pm 1.95$ & $3.44 \pm 1.18$ \\
\hline Tonetti et al. (PPF + EMD) 2002 & UK & 83 & 83 & No antibiotics & $3.1 \pm 1.5$ & $3.9 \pm 1.7$ & - \\
\hline Zucchelli et al. (PPF + EMD) 2002 & Italy & 30 & 30 & $\begin{array}{c}\text { Amoxicillin } 875 \mathrm{mg}+\text { clavulanic } 125 \mathrm{mg} \text { q.d } \\
\text { for } 7 \text { days }\end{array}$ & $4.2 \pm 0.9$ & $5.1 \pm 0.7$ & - \\
\hline Francetti et al. (PPF + EMD) 2005 & Italy & 64 & 82 & $\begin{array}{l}\text { Amoxicillin } 875 \mathrm{mg}+\text { clavulanic } 125 \mathrm{mg} \text { b.i.d. } \\
\text { for } 5 \text { days }\end{array}$ & $3.51 *$ & $4.02 *$ & - \\
\hline Wachtel et al. (PPF + EMD) 2003 & Germany & 11 & 26 & No antibiotics & $3.6 \pm 1.6$ & $3.9 \pm 1.4$ & - \\
\hline Grusovin et al. (PPF + EMD) 2009 & UK & 15 & 15 & No antibiotics & $3.4 \pm 1.1$ & $4.2 \pm 1.6$ & $2.5 \pm 1.2$ \\
\hline
\end{tabular}


Table 1. Cont.

\begin{tabular}{|c|c|c|c|c|c|c|c|}
\hline Author/Treatment & Country & $\begin{array}{c}\mathbf{N}^{\circ} \\
\text { Patients }\end{array}$ & $\begin{array}{c}\mathbf{N}^{\circ} \\
\text { Defects }\end{array}$ & Antibiotic Regimen & CAL Gain & $\begin{array}{c}\text { PPD } \\
\text { Reduction }\end{array}$ & Bone Gain \\
\hline \multicolumn{8}{|l|}{ EMD } \\
\hline Rosing et al. (PPF + EMD) 2005 & Norway & 14 & 14 & Penicillin $500 \mathrm{mg}$ t.i.d for 5 days & $\S$ & $\S$ & $\S$ \\
\hline Bokan et al. (PPF + EMD) 2006 & Germany & 19 & 19 & Doxycycline $100 \mathrm{mg}$ q.d. for 7 days & $3.7 \pm 1$ & $3.9 \pm 1.3$ & - \\
\hline Aslan et al. (PPF + EMD) 2020 & Turkey & 15 & 15 & Doxycycline $100 \mathrm{mg}$ b.i.d. for 7 days & $6.3 \pm 2.5$ & $6.5 \pm 2.65$ & - \\
\hline Mazzonetto et al. (PPF + EMD) 2020 & Brazil & 20 & 20 & No antibiotics & $2.4 \pm 1$ & $2.3 \pm 1.2$ & $1.24 \pm 1.14$ \\
\hline \multicolumn{8}{|l|}{ EMD + graft } \\
\hline Paolantonio et al. (PPF + EMD + AB) 2020 & Italy & 22 & 22 & $\begin{array}{l}\text { Amoxicillin } 875 \mathrm{mg}+\text { clavulanic } 125 \mathrm{mg} \text { b.i.d. } \\
\text { for } 6 \text { days }\end{array}$ & $3.29 \pm 0.85$ & $3.96 \pm 1.04$ & $2.67 \pm 1.06$ \\
\hline Guida et al. (OFD + EMD + AB) 2007 & Italy & 13 & 14 & Amoxicillin 875 + clavulanic 125 b.i.d. for 6 days & $4.9 \pm 1.8$ & $5.1 \pm 1.7$ & $4.3 \pm 1.3$ \\
\hline Yilmaz et al. (OFD + EMD + AB) 2010 & Turkey & 20 & 20 & Amoxicillin $500 \mathrm{mg}$ t.i.d. for 7 days & $4.2 \pm 1.1$ & $5.6 \pm 0.9$ & $3.9 \pm 1$ \\
\hline Sculean et al. (OFD + EMD + BG) $2005 b$ & Netherlands/Poland & 15 & 15 & No antibiotics & $3.2 \pm 1.7$ & $4.2 \pm 1.4$ & - \\
\hline Sculean et al. (OFD + EMD + BG) $2002 b$ & Germany & 14 & 14 & Amoxicillin $500 \mathrm{mg}$ t.i.d. for 7 days & $\S$ & $\S$ & - \\
\hline Ghezzi et al. (PPF + EMD + DBBM) 2016 & Italy & 10 & 10 & Amoxicillin 875 + clavulanic 125 b.i.d. for 7 days & $4.4 \pm 1.17$ & $4.9 \pm 1.2$ & - \\
\hline Sculean et al. (OFD + EMD + DBBM) 2002a & Germany & 12 & 12 & Amoxicillin $500 \mathrm{mg}$ t.i.d. for 7 days & $4.7 \pm 1.9$ & $5.7 \pm 1.5$ & - \\
\hline Iorio-Siciliano et al. (OFD + EMD + DBBM) 2014 & Italy & 20 & 20 & No antibiotics & $3.8 \pm 1.6$ & $4.6 \pm 1.9$ & - \\
\hline Döri et al. (OFD + EMD + DBBM) 2005 & Hungary & 12 & 12 & Amoxicillin $500 \mathrm{mg}$ t.i.d. for 7 days & $4.3 \pm 0.8$ & $4.8 \pm 0.9$ & - \\
\hline Döri et al. (OFD + EMD + DBBM) 2008b & Hungary & 13 & 13 & Amoxicillin $500 \mathrm{mg}$ t.i.d. for 7 days & $5 \pm 0.9$ & $5.9 \pm 1.3$ & - \\
\hline Aspriello et al. (OFD + EMD + DFDBA) 2011 & Italy & 28 & 28 & \multirow{2}{*}{$\begin{array}{l}\text { Ceftibuten } 400 \mathrm{mg} \text { q.d. for } 6 \text { day } \\
\text { Amoxicillin } 1 \mathrm{~g} \text { pre-operative and } 2 \mathrm{~g} \text { q.d. } \\
\text { for two days }\end{array}$} & $4^{*}$ & $5 *$ & $4 *$ \\
\hline Abu-ta et al. (OFD + EMD + DFDBA) 2016 & Palestine & 20 & 20 & & $4.3 \pm 1.1$ & $3.4 \pm 1.2$ & - \\
\hline Abu-ta et al. (OFD + EMD + DFDBA) 2016 & Palestine & 20 & 20 & \multirow{3}{*}{$\begin{array}{c}\text { No antibiotics } \\
\text { Amoxicillin } 500 \text { mg t.i.d. for } 7 \text { days } \\
\text { Amoxicillin } 750 \text { or Clindamycin } 300 \mathrm{mg} \text { t.i.d. } \\
\text { for } 7 \text { days }\end{array}$} & $4.1 \pm 1.4$ & $3.7 \pm 1.3$ & - \\
\hline Döri et al. (OFD + EMD + bTCP) 2005 & Hungary & 12 & 12 & & $4.1 \pm 0.8$ & $4.6 \pm 0.8$ & - \\
\hline Losada et al. (OFD + EMD + HA/bTCP) 2016 & Spain & 21 & 21 & & $2.38 \pm 2.17$ & $3.14 \pm 1.95$ & $2.71 \pm 1.79$ \\
\hline Meyle et al. (OFD + EMD + HA/bTCP) 2011 & Germany & 38 & 38 & No antibiotics & $1.69 \pm 2.1$ & $2.8 \pm 2.1$ & $2.65 \pm 1.9$ \\
\hline Pietruska et al. (OFD + EMD + HA/bTCP) 2011 & Pietruska & 12 & 12 & Amoxicillin $500 \mathrm{mg}$ t.i.d. for 7 days & $\S$ & $\S$ & $\begin{array}{c}2.00+1.0 \\
-\end{array}$ \\
\hline Bokan et al. (PPF + EMD + bTCP) 2006 & Germany & 19 & 19 & Doxycycline $100 \mathrm{mg}$ q.d. for 7 days & $4 \pm 1$ & $4.1 \pm 1.2$ & - \\
\hline Lee et al. (OFD + EMD + DPBM) 2020 & Korea & 20 & 20 & Amoxicillin $500 \mathrm{mg}$ t.i.d. for 5 days & $\S$ & $\S$ & - \\
\hline \multicolumn{8}{|l|}{ GTR-NR } \\
\hline Silvestri et al. (OFD + GTR-NR) 2000 & Italy & 10 & 10 & Amoxicillin 875 + clavulanic 125 b.i.d. for 7 days & $4.8 \pm 1.6$ & $5.9 \pm 1.1$ & - \\
\hline Mora et al. (OFD + GTR-NR) 1996 & France & 10 & 10 & Tetracycline 500 mg q.d. 8 days & $3.85 \pm 0.9$ & $5.35 \pm 1.1$ & $2.95 \pm 1.3$ \\
\hline Crea et al. (OFD + GTR-NR) 2008 & Italy & 20 & 20 & Amoxicillin $500 \mathrm{mg}$ b.i.d. for 7 days & $2 \pm 1.1$ & $3.2 \pm 1.1$ & $2.7 \pm 1.2$ \\
\hline Iorio-Siciliano et al. (OFD + GTR-NR) 2011 & Italy & 20 & 20 & No antibiotics & $4.1 \pm 1.4$ & $5.5 \pm 1$ & - \\
\hline
\end{tabular}


Table 1. Cont.

\begin{tabular}{|c|c|c|c|c|c|c|c|}
\hline Author/Treatment & Country & $\begin{array}{c}\mathbf{N}^{\circ} \\
\text { Patients }\end{array}$ & $\begin{array}{c}\mathbf{N}^{\circ} \\
\text { Defects }\end{array}$ & Antibiotic Regimen & CAL Gain & $\begin{array}{c}\text { PPD } \\
\text { Reduction }\end{array}$ & Bone Gain \\
\hline \multicolumn{8}{|l|}{ GTR-NR } \\
\hline Silvestri et al. (OFD + GTR-NR) 2003 & Italy & 49 & 49 & Amoxicillin 875 + clavulanic 125 b.i.d. for 7 days & $4.3 \pm 1.9$ & $5.6 \pm 1.5$ & - \\
\hline Pontoriero et al. (OFD + GTR-NR) 1999 & Sweden & 10 & 10 & 3G amoxicillin $1 \mathrm{~h}$ before surgery & $2.9^{*}$ & $4.7 *$ & - \\
\hline Christgau et al. (OFD + GTR-NR) 1997 & Germany & 10 & 10 & Doxycycline $100 \mathrm{mg}$ q.d. for 10 days & $3.7 \pm 3$ & $3.9 \pm 2.3$ & - \\
\hline Zybutz et al. (OFD + GTR-NR) 2000 & USA & 14 & 14 & $\begin{array}{l}\text { The day of surgery. Molecule } \\
\text { and dosage not specified }\end{array}$ & $2.4 \pm 0.8$ & $3.1 \pm 1.2$ & $2.2 \pm 1.7$ \\
\hline Zucchelli et al. (PPF + GTR-NR) 2002 & Italy & 30 & 30 & Amoxicillin 875 + clavulanic 125 q.d. for 7 days & $4.9 \pm 1.6$ & $6.5 \pm 1.6$ & - \\
\hline Mayfield et al. (OFD + GTR-R) 1998 & Sweden & 20 & 20 & No antibiotics & $1.5 \pm 1.9$ & $2.9 \pm 1.8$ & $0.6 \pm 1.2$ \\
\hline Paolantonio et al. (OFD + GTR-R) 2008 & Italy & 17 & 17 & Ampicillin $1 \mathrm{~g}$ b.i.d. for 7 days & $3.1 *$ & $5.2 *$ & $2.4^{*}$ \\
\hline Loos et al. (OFD + GTR-R) 2002 & Netherlands & 13 & 13 & $\begin{array}{l}\text { Amoxicillin } 375 \mathrm{mg} \text { and metronidazole } 250 \mathrm{mg} \text { t.i.d. } \\
\text { 8 days }\end{array}$ & $1.3^{*}$ & - & - \\
\hline Loos et al. (OFD + GTR-R) 2002 & Netherlands & 12 & 12 & No antibiotics & $1.5^{*}$ & - & - \\
\hline Blumenthal et al. (OFD + GTR-R) 1990 & USA & 15 & 15 & Tetracycline $250 \mathrm{mg}$ q.d. for 10 days & $1.17 \pm 0.1$ & $1.99 \pm 0.3$ & $1.83 \pm 0.2$ \\
\hline Pontoriero et al. (OFD + GTR-R) 1999 & Sweden & 10 & 10 & 3G amoxicillin $1 \mathrm{~h}$ before surgery & $3 *$ & $4.1 *$ & - \\
\hline Pontoriero et al. (OFD + GTR-R) 1999 & Sweden & 10 & 10 & 3G amoxicillin $1 \mathrm{~h}$ before surgery & $3.4^{*}$ & $4.8^{*}$ & - \\
\hline Sanz et al. (OFD + GTR-R) 2004 & Spain & 32 & 32 & No antibiotics & $2.5 \pm 1.9$ & $3.3 \pm 1.5$ & - \\
\hline Christgau et al. (OFD + GTR-R) 1997 & Germany & 10 & 10 & Doxycycline $100 \mathrm{mg}$ q.d. for 10 days & $3.8 \pm 1.9$ & $4 \pm 1.4$ & - \\
\hline Zybutz et al. (OFD + GTR-R) 2000 & USA & 15 & 15 & $\begin{array}{l}\text { The day of surgery. Molecule } \\
\text { and dosage not specified }\end{array}$ & $2.4 \pm 1.9$ & $3.3 \pm 2.1$ & $2.4 \pm 3.7$ \\
\hline Trejo et al. (OFD + GTR-R) 2000 & USA & 14 & 14 & Doxycycline $100 \mathrm{mg}$ q.d. for 10 days & $3.27 \pm 1.1$ & $4.12 \pm 0.84$ & $5.35 \pm 2.91$ \\
\hline Paolantonio et al. (OFD + GTR-R) 2002 & Italy & 17 & 17 & Ampicillin $1 \mathrm{~g}$ b.i.d. for 7 days & $4 \pm 1.27$ & $5.58 \pm 1$ & $3.82 \pm 1.28$ \\
\hline Mengel et al. (OFD + GTR-R) 2003 & Germany & - & 22 & No antibiotics & $3.4 \pm 2.3$ & $4 \pm 2.1$ & - \\
\hline Tonetti et al. (PPF + GTR-R) 1998 & Switzerland & 69 & 69 & Doxycycline $100 \mathrm{mg}$ b.i.d. for 7 days & $3.04 \pm 1.64$ & $4.03 \pm 1.81$ & - \\
\hline Cortellini et al. (PPF + GTR-R) 2001 & Italy & 55 & 55 & Doxycycline $200 \mathrm{mg}$ q.d. for 7 days & $3.5 \pm 2.1$ & $4.4 \pm 2.4$ & - \\
\hline Stravopolous et al. (PPF + GTR-R) 2003 & Denmark & 14 & 14 & $\begin{array}{l}\text { Amoxicillin } 750 \mathrm{mg}+\text { metronidazole } 250 \mathrm{mg} \text { q.d. } \\
\text { for } 5 \text { days }\end{array}$ & $2.9 *$ & $3.9 *$ & $3.1 *$ \\
\hline \multicolumn{8}{|l|}{ Graft + GTR-R } \\
\hline Sculean et al. (OFD + DBBM + GTR-R) 2003 & Germany & 14 & 14 & Amoxicillin $500 \mathrm{mg}$ t.i.d. for 7 days & $4 \pm 1.3$ & $5.3 \pm 1.6$ & - \\
\hline Sculean et al. (OFD + DBBM + GTR-R) 2005a & Netherlands & 16 & 16 & No antibiotics & $4.1 \pm 0.9$ & $5.4 \pm 0.9$ & - \\
\hline Tonetti et al. (OFD + DBBM + GTR-R) 2004 & UK & 61 & 61 & Doxycycline $200 \mathrm{mg}$ q.d. for 7 days & $3.3 \pm 1.7$ & $3.7 \pm 1.8$ & - \\
\hline $\begin{array}{l}\text { Iorio-Siciliano et al. } \\
(\text { OFD + DBBM + GTR-R) } 2014\end{array}$ & Italy & 20 & 20 & No antibiotics & $3.7 \pm 1.2$ & $4.4 \pm 1.7$ & - \\
\hline
\end{tabular}


Table 1. Cont.

\begin{tabular}{|c|c|c|c|c|c|c|c|}
\hline Author/Treatment & Country & $\begin{array}{c}\mathbf{N}^{\circ} \\
\text { Patients }\end{array}$ & $\begin{array}{c}\mathbf{N}^{\circ} \\
\text { Defects }\end{array}$ & Antibiotic Regimen & CAL Gain & $\begin{array}{c}\text { PPD } \\
\text { Reduction }\end{array}$ & Bone Gain \\
\hline \multicolumn{8}{|l|}{ Graft + GTR-R } \\
\hline Döri et al. (OFD + DBBM + GTR-R) 2007b & Hungary & 12 & 12 & Amoxicillin $500 \mathrm{mg}$ t.i.d. for 7 days & $4.6 \pm 0.8$ & $5.7 \pm 1.2$ & - \\
\hline Paolantonio et al. (OFD + DBBM + GTR-R) 2002 & Italy & 17 & 17 & Ampicillin $1 \mathrm{~g}$ b.i.d. for 7 days & $5.05 \pm 1.56$ & $5.76 \pm 1.6$ & $5.23 \pm 1.3$ \\
\hline Stravopolous et al. (PPF + DBBM + GTR-R) 2003 & Denmark & 15 & 15 & $\begin{array}{l}\text { Amoxicillin } 750 \mathrm{mg}+\text { metronidazole } 250 \mathrm{mg} \text { q.d. } \\
\text { for } 5 \text { days }\end{array}$ & 2.5 * & $3.8 *$ & $2.8^{*}$ \\
\hline Ghezzi et al. (OFD + DBBM + GTR-R) 2016 & Italy & 10 & 10 & Amoxicillin 875 + clavulanic 125 b.i.d. for 7 days & $4 \pm 1.82$ & $4.7 \pm 2.36$ & - \\
\hline Pietruska et al. (PPF + DBBM + GTR-R) 2020 & Poland & 21 & 21 & Amoxicillin 2 g q.d. for 7 days & $3.6 \pm 1.6$ & $3.8 \pm 1.3$ & $3.2 \pm 2.1$ \\
\hline Pietruska et al. (PPF + DBBM + GTR-R) 2020 & Poland & 20 & 20 & No antibiotics & $2.7 \pm 1.6$ & $3.3 \pm 1.7$ & $2.5 \pm 1.9$ \\
\hline Kim et al. (OFD + DFDBA + CS barrier) 1998 & Korea & 13 & 13 & Tetracycline $250 \mathrm{mg}$ q.d. for 7 days & $2.9 \pm 0.8$ & $4.3 \pm 0.5$ & $2.9 \pm 1.4$ \\
\hline Trejo et al. (OFD + DFDBA + GTR-R) 2000 & USA & 16 & 16 & Doxycycline $100 \mathrm{mg}$ q.d. for 10 days & $2.29 \pm 0.61$ & $3.37 \pm 1.16$ & $4.73 \pm 1.18$ \\
\hline Christgau et al. (OFD + bTCP + GTR-R) 2006 & Germany & 25 & 25 & Doxycycline $100 \mathrm{mg}$ q.d. for 19 days & $5.2 \pm 1.6$ & $6 \pm 1.1$ & - \\
\hline Orsini et al. (OFD + AB + CSM) 2008 & Italy/Spain & 12 & 12 & $\begin{array}{l}\text { Antibiotic for } 1 \text { week. } \\
\text { The molecule and dosage were not specified }\end{array}$ & $2.6 \pm 1.2$ & $3.3 \pm 1.6$ & - \\
\hline Orsini et al. (OFD + AB + GTR-R) 2008 & Italy/Spain & 12 & 12 & $\begin{array}{l}\text { Antibiotic for } 1 \text { week. } \\
\text { The molecule and dosage were not specified }\end{array}$ & $2.4 \pm 1.1$ & $4.2 \pm 1.2$ & - \\
\hline Cetinkaya et al. (OFD + BG + GTR-R) 2014 & Turkey & 11 & 11 & No Antibiotics & $2.64 \pm 1.12$ & $3.45 \pm 0.93$ & $3 \pm 1.48$ \\
\hline Yamamiya et al. (OFD + HA + HCPC) 2008 & Japan & 15 & 15 & Cefaclor $750 \mathrm{mg}$ q.d. for 5 days & $2.7 \pm 1.3$ & $4.3 \pm 1.1$ & $3.2 \pm 1.1$ \\
\hline $\begin{array}{c}\text { Nygaard-Østby et al. (OFD + AB + GTR-NR) } \\
2010\end{array}$ & Norway & 13 & 13 & Amoxicillin 500 mg b.i.d. for 10 days & $3.8 \pm 0.5$ & $4.2 \pm 0.5$ & $3.9 \pm 0.8$ \\
\hline Döri et al. (OFD + bTCP + GTR-NR) 2008a & Hungary & 14 & 14 & Amoxicillin $500 \mathrm{mg}$ t.i.d. for 7 days & $3.9 \pm 0.2$ & $5.4 \pm 0.7$ & - \\
\hline Döri et al. (OFD + DBBM + GTR-NR) 2007a & Hungary & 15 & 15 & Amoxicillin $500 \mathrm{mg}$ t.i.d. for 7 days & $4.6 \pm 1.1$ & $5.5 \pm 1.7$ & - \\
\hline \multicolumn{8}{|l|}{ Graft } \\
\hline Nygaard-Østby et al. (OFD + AB) 2010 & Norway & 13 & 13 & Amoxicillin $500 \mathrm{mg}$ b.i.d. for 10 days & $2.2 \pm 0.7$ & $2.7 \pm 0.5$ & $1.3 \pm 0.9$ \\
\hline Leknes et al. (OFD + BG) 2009 & Norway & 13 & 13 & No Antibiotics & $1.2 \pm 0.2$ & $2.6 \pm 1.1$ & - \\
\hline Mengel et al. (OFD + BG) 2003 & Germany & - & 20 & No Antibiotics & $2.8 \pm 1.8$ & $3.8 \pm 1.9$ & - \\
\hline Sculean et al. (OFD + BG) 2002b & Germany & 14 & 14 & Amoxicillin $500 \mathrm{mg}$ t.i.d. for 7 days & $\S$ & $\S$ & - \\
\hline Yassibag-Berkman et al. (OFD + bTCP) 2007 & Turkey & - & 10 & Amoxicillin 875 + clavulanic 125 b.i.d. for 5 days & $2.5 *$ & $4.1 *$ & - \\
\hline Pietruska et al. (OFD + HA) 2012 & Poland & 15 & 15 & Amoxicillin $1 \mathrm{~g}$ b.i.d. for 7 days & $2 \pm 2.7$ & $2.9 \pm 2.5$ & $1.9 \pm 1.5$ \\
\hline Al Machot et al. (OFD + HA) 2014 & Germany & 19 & 19 & No Antibiotics & $2.1 \pm 1.6$ & $3.2 \pm 1.8$ & $1.6 \pm 1.2$ \\
\hline Kasaj et al. (OFD + HA/p-15) 2008 & Germany & 13 & 13 & No antibiotics & $3.9 \pm 1.7$ & $4.3 \pm 1.3$ & - \\
\hline Okuda et al. (OFD + HA + saline) 2005 & Japan & 35 & 35 & Cefaclor $750 \mathrm{mg}$ q.d. for 5 days & $2 \pm 1.2$ & $3.7 \pm 2$ & $2.7 \pm 1.6$ \\
\hline De Leonardis et al. (PPF + HA/bTCP) 2013 & Italy & 34 & 34 & Amoxicillin 2 g q.d. for 6 days & $3.63 \pm 0.91$ & $4.25 \pm 0.63$ & $3.35 \pm 0.8$ \\
\hline
\end{tabular}


Table 1. Cont.

\begin{tabular}{|c|c|c|c|c|c|c|c|}
\hline Author/Treatment & Country & $\begin{array}{c}\mathbf{N}^{\circ} \\
\text { Patients }\end{array}$ & $\begin{array}{c}\mathbf{N}^{\circ} \\
\text { Defects }\end{array}$ & Antibiotic Regimen & CAL Gain & $\begin{array}{c}\text { PPD } \\
\text { Reduction }\end{array}$ & Bone Gain \\
\hline $\begin{array}{c}\text { Graft } \\
\text { Scabbia et al. } \\
(\mathrm{PPF}+\mathrm{HA} / \text { collagen } / \text { chondroitin sulfate }) 2004\end{array}$ & Italy & 13 & 13 & No Antibiotics & $2.9 \pm 1.9$ & $4.2 \pm 2.1$ & $2.5 \pm 1.4$ \\
\hline Slotte et al. (OFD + DBBM) 2012 & Sweden & 16 & 16 & $\begin{array}{l}\text { Phenoxymethylpenicillin } 2 \mathrm{~g} \\
\text { or clindamycin } 300 \mathrm{mg} \text { b.i.d. for } 7 \text { days }\end{array}$ & $2.3 \pm 0.8$ & $3.2 \pm 0.7$ & $3.4 \pm 2.3$ \\
\hline Sculean et al. (OFD + DBBM) 2002a & Germany & 12 & 12 & Amoxicillin 500 mg t.i.d. for 7 days & $4.9 \pm 2.1$ & $6.5 \pm 2$ & - \\
\hline Döri et al. (OFD + DBBM) 2009 & Hungary & 15 & 15 & Amoxicillin $500 \mathrm{mg}$ t.i.d. for 7 days & $4.7 \pm 1.6$ & $5.3 \pm 1.7$ & - \\
\hline Scabbia et al. (PPF + DBBM) 2004 & Italy & 11 & 11 & No Antibiotics & $4 \pm 2.4$ & $4.4 \pm 2.3$ & $3.1 \pm 1.8$ \\
\hline Agarwal et al. (OFD + DFDBA) 2014 & India & 24 & 24 & Amoxicillin $500 \mathrm{mg}$ t.i.d. for 7 days & $2.4 \pm 0.61$ & $3.65 \pm 0.52$ & $2.37 \pm 0.47$ \\
\hline Piemontese et al. (OFD + DFDBA) 2008 & Italy & 30 & 30 & Ceftibuten 400 mg q.d. for 5 days & $2.4 \pm 2.2$ & $3.5 \pm 1.9$ & $2.6 \pm 1.8$ \\
\hline Agarwal et al. (OFD + DFDBA) 2015 & India & 30 & 30 & Amoxicillin $500 \mathrm{mg}$ t.i.d. for 7 days & $2.61 \pm 0.68$ & $3.6 \pm 0.51$ & $2.49 \pm 0.64$ \\
\hline Aspriello et al. (OFD + DFDBA) 2011 & Italy & 28 & 28 & Ceftibuten 400 mg q.d. for 6 days & $3.25 *$ & $4 *$ & $3.5^{*}$ \\
\hline Blumenthal et al. (OFD + AAA) 1990 & USA & - & 14 & Tetracycline $250 \mathrm{mg}$ q.d. for 10 days & $1.43 \pm 0.1$ & $2.03 \pm 0.1$ & $2.06 \pm 0.1$ \\
\hline Shirakata et al. (OFD + CPC) 2008 & Japan & 15 & 15 & The molecule and dosage were not specified. & $2.3 \pm 1$ & $3.4 \pm 1.2$ & $1.2 \pm 0.8$ \\
\hline Paolantonio et al. (OFD + CS) 2008 & Italy & 17 & 17 & Ampicillin $1 \mathrm{~g}$ b.i.d. for 7 days & $2.7^{*}$ & $4.4^{*}$ & $2.3 *$ \\
\hline Lee et al. (OFD + DPBM) 2020 & Korea & 22 & 22 & Amoxicillin $500 \mathrm{mg}$ t.i.d. for 5 days & $\S$ & $\S$ & - \\
\hline \multicolumn{8}{|l|}{ PRP/PRF } \\
\hline Thorat et al. (OFD + PRF) 2017 & India & 15 & 15 & $\begin{array}{l}\text { Amoxicillin } 500 \mathrm{mg}+ \\
\text { metronidazole } 400 \mathrm{mg} \text { q.d. for } 7 \text { days }\end{array}$ & $4 \pm 0.63$ & $4 \pm 0.63$ & - \\
\hline Patel et al. (OFD + PRF) 2017 & India & 13 & 13 & Amoxicillin 500 mg t.i.d. for 7 days & $3.7 \pm 0.67$ & $4.2 \pm 1.69$ & - \\
\hline \multicolumn{8}{|l|}{ Graft + PRP/PRF } \\
\hline Döri et al. (OFD + DBBM + PRP) 2009 & Hungary & 15 & 15 & Amoxicillin $500 \mathrm{mg}$ t.i.d. for 7 days & $4.6 \pm 1.7$ & $5.2 \pm 1.6$ & - \\
\hline Qiao et al. (OFD + DBBM + PRP(CGF)) 2016 & China & - & 15 & Amoxicillin $500 \mathrm{mg}$ t.i.d. for 7 days & $3.7 \pm 1.3$ & $4.2 \pm 1.3$ & $3.3 \pm 1.5$ \\
\hline Agarwal et al. (OFD + DFDBA + PRF) 2015 & India & 30 & 30 & Amoxicillin $500 \mathrm{mg}$ t.i.d. for 7 days & $3.73 \pm 0.74$ & $4.15 \pm 0.84$ & $3.5 \pm 0.67$ \\
\hline Agarwal et al. (OFD + DFDBA + PRP) 2014 & India & 24 & 24 & Amoxicillin $500 \mathrm{mg}$ t.i.d. for 7 days & $3.15 \pm 0.5$ & $3.64 \pm 0.63$ & $3.02 \pm 0.5$ \\
\hline Piemontese et al. (OFD + DFDBA + PRP) 2008 & Italy & 30 & 30 & Ceftibuten 400 mg q.d. for 5 days & $3.6 \pm 1.8$ & $4.6 \pm 1.3$ & $3.3 \pm 1.5$ \\
\hline Okuda et al. (OFD + HA + PRP) 2005 & Japan & 35 & 35 & Cafaclor 750 mg q.d. for 5 days & $3.4 \pm 1.7$ & $4.7 \pm 1.6$ & $3.5 \pm 1.5$ \\
\hline Yamamiya et al. (OFD + HA + PRP) 2008 & Japan & 15 & 15 & Cafaclor $750 \mathrm{mg}$ q.d. for 5 days & $3.9 \pm 1.6$ & $4.8 \pm 1.1$ & $4.9 \pm 1.2$ \\
\hline $\begin{array}{l}\text { Yassibag-Berkman et al. } \\
\text { (OFD + bTCP + PRP) } 2007\end{array}$ & Turkey & - & 10 & $\begin{array}{c}\text { Amoxicillin } 875 \text { + clavulanic } \\
125 \text { b.i.d. for } 5 \text { days }\end{array}$ & $2.1 *$ & $3.6^{*}$ & - \\
\hline Paolantonio et al. (PPF + AB + 1-PRF) 2020 & Italy & 22 & 22 & $\begin{array}{l}\text { Amoxicillin } 875 \mathrm{mg}+\text { clavulanic } \\
125 \mathrm{mg} \text { b.i.d. for } 6 \text { days }\end{array}$ & $3.43 \pm 0.74$ & $4.21 \pm 1.1$ & $2.92 \pm 0.71$ \\
\hline
\end{tabular}


Table 1. Cont.

\begin{tabular}{|c|c|c|c|c|c|c|c|}
\hline Author/Treatment & Country & $\begin{array}{c}\mathbf{N}^{\circ} \\
\text { Patients }\end{array}$ & $\begin{array}{c}\mathbf{N}^{\circ} \\
\text { Defects }\end{array}$ & Antibiotic Regimen & CAL Gain & $\begin{array}{c}\text { PPD } \\
\text { Reduction }\end{array}$ & Bone Gain \\
\hline Other Combinations & & & & & & & \\
\hline Blumenthal et al. (OFD + AAA + CG) 1990 & USA & - & 12 & Tetracycline $250 \mathrm{mg}$ q.d. for 10 days & $1.88 \pm 0.2$ & $2.61 \pm 0.1$ & $2.88 \pm 0.2$ \\
\hline $\begin{array}{c}\text { Blumenthal et al. } \\
(\text { OFD + AAA + CG + GTR-R) } 1990\end{array}$ & USA & - & 15 & Tetracycline $250 \mathrm{mg}$ q.d. for 10 days & $2.01 \pm 0.1$ & $2.73 \pm 0.1$ & $3.71 \pm 0.1$ \\
\hline $\begin{array}{c}\text { Döri et al. } \\
(\mathrm{OFD}+\mathrm{bTCP}+\text { GTR-NR + PRP) 2008a }\end{array}$ & Hungary & 14 & 14 & Amoxicillin $500 \mathrm{mg}$ t.i.d. for 7 days & $4.1 \pm 0.7$ & $5.8 \pm 0.6$ & - \\
\hline $\begin{array}{c}\text { Christgau et al. } \\
(\mathrm{OFD}+\mathrm{bTCP}+\text { GTR-R + PRP) } 2006\end{array}$ & Germany & 25 & 25 & Doxycycline $100 \mathrm{mg}$ q.d. for 19 days & $5 \pm 1.5$ & $6.3 \pm 1.2$ & - \\
\hline $\begin{array}{c}\text { Yassibag-Berkman et al. } \\
\text { (OFD + bTCP + PRP + GTR-R) } 2007\end{array}$ & Turkey & - & 10 & Amoxicillin 875 + clavulanic 125 b.i.d. for 5 days & $2.4^{*}$ & $4^{*}$ & - \\
\hline Döri et al. (OFD + DBBM + EMD + PRP) 2008b & Hungary & 13 & 13 & Amoxicillin $500 \mathrm{mg}$ t.i.d. for 7 days & $4.8 \pm 1.3$ & $5.8 \pm 1.8$ & - \\
\hline $\begin{array}{c}\text { Döri et al. } \\
(\text { OFD + DBBM + GTR-NR + PRP) 2007a }\end{array}$ & Hungary & 15 & 15 & Amoxicillin $500 \mathrm{mg}$ t.i.d. for 7 days & $4.5 \pm 1.1$ & $5.5 \pm 1.3$ & - \\
\hline Döri et al. (OFD + DBBM + GTR-R + PRP) 2007b & Hungary & 12 & 12 & Amoxicillin $500 \mathrm{mg}$ t.i.d. for 7 days & $4.7 \pm 1.1$ & $5.5 \pm 1.2$ & - \\
\hline Minabe et al. (OFD + EMD + GTR-R) 2002 & Japan & - & 23 & Cefaclor $750 \mathrm{mg}$ q.d. for 4 days & $3 \pm 1.3$ & $4.3 \pm 1.6$ & - \\
\hline Sipos et al. (OFD + EMD + GTR-R) 2005 & Netherlands & 11 & 12 & No antibiotics & $1.65 \pm 1.29$ & $3.02 \pm 1.55$ & $1.58 \pm 1.92$ \\
\hline Cetinkaya et al. (OFD + PP + GTR-R) 2014 & Turkey & 11 & 11 & No antibiotics & $2.36 \pm 0.92$ & $2.91 \pm 0.94$ & $3.45 \pm 1.81$ \\
\hline Aoki et al. (PPF + DBBM + rhFGF-2) 2020 & Japan & 20 & 20 & Amoxicillin $750 \mathrm{mg}$ or cefdinir $300 \mathrm{mg}$ q.d. for 4 days & $3.11 \pm 1.46$ & $3.58 \pm 1.53$ & - \\
\hline Ferrarotti et al. (PPF + DPSCs) 2018 & Italy & 15 & 15 & Amoxicillin 875 + clavulanic 125, 1 g q.d. for 6 days & $4.5 \pm 1.9$ & $4.9 \pm 1.4$ & $3.9 \pm 1.5$ \\
\hline Aoki et al. (PPF + rhFGF-2) 2020 & Japan & 18 & 18 & Amoxicillin $750 \mathrm{mg}$ or cefdinir $300 \mathrm{mg}$ q.d. for 4 days & $3.35 \pm 1.28$ & $3.58 \pm 1.21$ & - \\
\hline Santana et al. (PPF + rhFGF-2/HyAc) 2015 & Brazil & 30 & 30 & $\begin{array}{l}\text { Doxycycline } 200 \mathrm{mg} \text { before surgery } \\
\text { and } 100 \mathrm{mg} \text { q.d. for } 19 \text { days }\end{array}$ & $4.8 \pm 0.2$ & $5.5 \pm 1.4$ & - \\
\hline
\end{tabular}

*: The standard deviation was not reported. \&. The authors reported baseline and follow-up data but not the difference. AAA: autolyzed antigen-extracted allogenic freeze-dried bone AB: autogenous bone; BG: bioactive glass; bTCP: Tricalcium Phosphate; CG: microfibrillar collagen gel; CPC: calcium phosphate bone cement; CS: calcium sulfate; CSM: calcium sulphate membrane; DBBM: demineralized bovine bone matrix; DFDBA: demineralized freeze-dried bone allograft; DPBM: demineralized porcine bone matrix; DPSCs: dental pulp stem cells; EMD: enamel matrix derivative; GTR-NR: guided tissue regeneration using a non-resorbable membrane; GTR-R: GTR using a resorbable membrane; HA: hydroxyapatite; HA/P-15: hydroxyapatite matrix/cell-binding peptide; HCPC: human cultured periosteum used in sheet as a membrane; HyAc: hyaluronic acid; 1-PRF: leukocyte platelet-rich fibrin OFD: open flap debridement; PDGF: platelet-derived growth factor; PLA/PGA polylactide/polyglicolide copolymer as biomaterial; PP: platelet pellet; PPF: papilla preservation flap; PRF: platelet-rich fibrin; rhFGF-2: recombinant human fibroblast growth factor. 
Table 2. Characteristics of the treatment arms from included studies treating furcation defects.

\begin{tabular}{|c|c|c|c|c|c|c|c|c|}
\hline Author/Treatment & Country & $\begin{array}{c}\mathbf{N}^{\circ} \\
\text { Patient }\end{array}$ & $\begin{array}{c}\mathbf{N}^{\circ} \\
\text { Defect }\end{array}$ & Antibiotic Regimen & $\begin{array}{l}\text { V-CAL } \\
\text { Gain }\end{array}$ & $\begin{array}{l}\text { H-CAL } \\
\text { Gain }\end{array}$ & $\begin{array}{c}\text { PPD } \\
\text { Reduction }\end{array}$ & $\begin{array}{l}\text { H-BL } \\
\text { Gain }\end{array}$ \\
\hline \multicolumn{9}{|l|}{ GTR-R } \\
\hline Blumenthal et al. (OFD + GTR-R) 1993 & USA & 12 & 12 & Amoxicillin $500 \mathrm{mg}$ t.i.d for 7 days & $1.83 \pm 1.47$ & $2.5 \pm 0.8$ & $3.08 \pm 1.68$ & $2.5 \pm 0.7$ \\
\hline Bouchard et al. (OFD + GTR-R) 1993 & France & 12 & 12 & Doxycycline $100 \mathrm{mg}$ q.d. for 14 days & $1.2 \pm 1.8$ & $1.5 \pm 1.5$ & $1.9 \pm 2$ & $1.5 \pm 1.1$ \\
\hline Maragos et al. (OFD + GTR-R) 2002 & USA & - & 11 & Doxycycline $100 \mathrm{mg}$ q.d. for 10 days & $1.4 \pm 0.4$ & - & - & $0.9 \pm 0.12$ \\
\hline Pruthi et al. (OFD + GTR-R) 2002 & Canada & 17 & 17 & $\begin{array}{l}\text { Doxycycline } 200 \mathrm{mg} \text { day of surgery } \\
\text { and } 100 \mathrm{mg} \text { q.d for } 13 \text { days }\end{array}$ & $1 \pm 1.22$ & - & $1.47 \pm 1.01$ & $0.41 \pm 0.71$ \\
\hline Wang et al. (OFD + GTR-R) 1994 & USA & 12 & 12 & Doxycycline $100 \mathrm{mg}$ q.d. for 14 days & $1.67 \pm 0.76$ & - & $2.8 \pm 1.4$ & - \\
\hline Garrett et al. (OFD + GTR-R) 1997 & USA & 66 & 66 & $\begin{array}{l}\text { Doxycycline } 100 \mathrm{mg} \text { b.i.d. the day } \\
\text { of surgery and q.d. for } 13 \text { days }\end{array}$ & $2 \pm 0.2$ & $2.1 \pm 0.4$ & $2.3 \pm 0.45$ & - \\
\hline Bouchard et al. (OFD + GTR-R) 1997 & France & 15 & 15 & $\begin{array}{l}\text { Amoxicillin }+ \\
\text { clavulanic } 1.5 \text { g q.d. for } 14 \text { days }\end{array}$ & $1.5 \pm 1.9$ & $2.5 \pm 1.6$ & $2.1 \pm 1.6$ & - \\
\hline Hugoson et al. (OFD + GTR-R) 1995 & Sweden & 38 & 38 & No Antibiotics & $0.4 \pm 1.5$ & $1.4 \pm 2.2$ & $2.2 \pm 1.4$ & - \\
\hline Karapataki et al. (OFD + GTR-R) 1999 & Sweden & 11 & 11 & No antibiotics & $1.1 \pm 1.2$ & $2.3 \pm 2.23$ & - & - \\
\hline Jepsen et al. (OFD + GTR-R) 2004 & Germany & 45 & 45 & No Antibiotics & - & - & - & $1.9 \pm 1.4$ \\
\hline \multicolumn{9}{|l|}{ GTR-NR } \\
\hline Bouchard et al. (OFD + GTR-NR) 1993 & France & 12 & 12 & Doxycycline $100 \mathrm{mg}$ q.d. for 14 days & $1.3 \pm 1.6$ & $2.8 \pm 1.3$ & $2.2 \pm 1.2$ & $2.2 \pm 1.4$ \\
\hline Pruthi et al. (OFD + GTR-NR) 2002 & Canada & 17 & 17 & $\begin{array}{l}\text { Doxycycline } 200 \mathrm{mg} \text { day of surgery } \\
\text { and } 100 \mathrm{mg} \text { q.d for } 13 \text { days }\end{array}$ & $0.47 \pm 1.81$ & - & $1.12 \pm 1.36$ & $0.41 \pm 0.62$ \\
\hline Garrett et al. (OFD + GTR-NR) 1997 & USA & 64 & 64 & $\begin{array}{l}\text { Doxycycline } 100 \mathrm{mg} \text { b.i.d. the day of } \\
\text { surgery and q.d. for } 13 \text { days }\end{array}$ & $1.6 \pm 0.2$ & $2.1 \pm 0.4$ & $2.1 \pm 0.45$ & - \\
\hline Bouchard et al. (OFD + GTR-NR) 1997 & France & 15 & 15 & $\begin{array}{l}\text { Amoxicillin }+ \\
\text { clavulanic } 1.5 \text { g q.d. for } 14 \text { days }\end{array}$ & $1.2 \pm 1.2$ & $2.7 \pm 1.2$ & $1.8 \pm 1.3$ & - \\
\hline Hugoson et al. (OFD + GTR-NR) 1995 & Sweden & 38 & 38 & No Antibiotics & $0.8 \pm 1.4$ & $2.2 \pm 2$ & $2 \pm 1$ & - \\
\hline Karapataki et al. (OFD + GTR-NR) 1999 & Sweden & 11 & 11 & No antibiotics & $0.5 \pm 1.2$ & $0.5 \pm 2.5$ & - & - \\
\hline Leite et al. (OFD + GTR-NR) 2013 * & Brazil & 12 & 12 & $\begin{array}{l}\text { Amoxicillin + clavulanic } 500 \mathrm{mg} \text { t.i.d } \\
\text { for } 10 \text { days starting the day before surgery }\end{array}$ & $0.02 \pm 1.32$ & - & $0.43 \pm 0.84$ & $1.36 \pm 1.57$ \\
\hline Leite et al. (OFD + GTR-NR) 2013 & Brazil & 12 & 12 & $\begin{array}{l}\text { Amoxicillin + clavulanic } 500 \mathrm{mg} \text { t.i.d } \\
\text { for } 10 \text { days starting the day before surgery }\end{array}$ & $0.24 \pm 1.15$ & - & $0.37 \pm 1.03$ & $1.18 \pm 1.08$ \\
\hline Villaça et al. (OFD + GTR-NR) 2004 & Brazil & 10 & 10 & $\begin{array}{l}\text { Amoxicillin + clavulanic } 500 \mathrm{mg} \text { t.i.d } \\
\text { for } 10 \text { days starting the day before surgery }\end{array}$ & $\S$ & - & $\S$ & $\S$ \\
\hline Villaça et al. (OFD + GTR-NR) 2004 † & Brazil & 10 & 10 & $\begin{array}{l}\text { Amoxicillin + clavulanic } 500 \mathrm{mg} \text { t.i.d } \\
\text { for } 10 \text { days starting the day before surgery }\end{array}$ & $\S$ & - & $\S$ & $\S$ \\
\hline
\end{tabular}


Table 2. Cont.

\begin{tabular}{|c|c|c|c|c|c|c|c|c|}
\hline Author/Treatment & Country & $\begin{array}{c}\mathbf{N}^{\circ} \\
\text { Patient }\end{array}$ & $\begin{array}{c}\mathbf{N}^{\circ} \\
\text { Defect }\end{array}$ & Antibiotic Regimen & $\begin{array}{l}\text { V-CAL } \\
\text { Gain }\end{array}$ & $\begin{array}{l}\text { H-CAL } \\
\text { Gain }\end{array}$ & $\begin{array}{c}\text { PPD } \\
\text { Reduction }\end{array}$ & $\begin{array}{l}\text { H-BL } \\
\text { Gain }\end{array}$ \\
\hline Combinations & & & & & & & & \\
\hline Maragos et al. (OFD + GTR-R + DFDBA) 2002 & USA & - & 14 & Doxycycline $100 \mathrm{mg}$ q.d. for 10 days & $2.6 \pm 0.3$ & - & - & $2.2 \pm 0.15$ \\
\hline Jaiswal et al. (OFD + GTR-R + DFDBA) 2013 & India & 10 & 10 & Amoxicillin $500 \mathrm{mg}$ t.i.d for 7 days & $0.85 \pm 0.31$ & - & $0.8 \pm 0.72$ & - \\
\hline de Leonardis et al. (OFD + GTR-R + DFDBA) 1999 & Italy & 12 & 12 & $\begin{array}{l}\text { Amoxicillin } 875 \mathrm{mg}+ \\
\text { clavulanic } 125 \mathrm{mg} \text { b.i.d. for } 7 \text { days }\end{array}$ & $2.3 \pm 0.85$ & - & $2.8 \pm 0.9$ & - \\
\hline Garrett et al. (CAF + GTR-R + DFDBA) 1990 & USA & 13 & 15 & $\begin{array}{l}\text { Penicillin or erythromycin } 250 \mathrm{mg} \text { q.i.d. } \\
\text { for } 14 \text { days }\end{array}$ & - & - & $2.2 \pm 0.3$ & $2 \pm 2.1$ \\
\hline Garrett et al. (OFD + DFDBA) 1990 & USA & 12 & 16 & $\begin{array}{l}\text { Penicillin or erythromycin } 250 \mathrm{mg} \text { q.i.d. } \\
\text { for } 14 \text { days }\end{array}$ & - & - & $2.6 \pm 1.13$ & $2.6 \pm 1.1$ \\
\hline Gantes et al. (OFD + DFDBA) 1991 & USA & 13 & 13 & Tetracycline $250 \mathrm{mg}$ q.i.d for 14 days & $\int$ & - & $\int$ & - \\
\hline Jaiswal et al. (OFD + GTR-R + DFDBA + EMD) 2013 & India & 10 & 10 & Amoxicillin $500 \mathrm{mg}$ t.i.d for 7 days & $2.12 \pm 1.07$ & - & $1.74 \pm 1$ & - \\
\hline de Santana et al. (CAF + GTR-R + HA) 1999 & USA & 15 & 15 & $\begin{array}{l}\text { Doxycycline } 200 \mathrm{mg} \text { day of surgery } \\
\text { and } 100 \mathrm{mg} \text { q.d for } 19 \text { days }\end{array}$ & $1.57 \pm 1.32$ & $2.13 \pm 1.52$ & $2.43 \pm 1.36$ & - \\
\hline Santana et al. (CAF + GTR-NR + HA) 2009 & Brazil & 30 & 30 & $\begin{array}{l}\text { Doxycycline } 200 \mathrm{mg} \text { day of surgery } \\
\text { and } 100 \mathrm{mg} \text { q.d for } 19 \text { days }\end{array}$ & $3.05 \pm 0.6$ & - & $3.56 \pm 0.6$ & - \\
\hline Jepsen et al. (OFD + EMD) 2004 & Germany & 45 & 45 & No Antibiotics & - & - & - & $2.6 \pm 1.8$ \\
\hline Queiroz et al. (OFD + EMD) 2016 & Brazil & 13 & 13 & No Antibiotics & $2.08 \pm 1.61$ & $2.77 \pm 0.93$ & $2.54 \pm 0.78$ & - \\
\hline Queiroz et al. (OFD + bTCP/HA) 2016 & Brazil & 14 & 14 & No Antibiotics & $2.29 \pm 1.27$ & $2.64 \pm 0.93$ & $2.36 \pm 1.01$ & - \\
\hline Queiroz et al. (OFD + bTCP /HA + EMD) 2016 & Brazil & 14 & 14 & No Antibiotics & $2.14 \pm 1.29$ & $2.93 \pm 0.83$ & $2.43 \pm 1.02$ & - \\
\hline
\end{tabular}

*: e-PTFE membrane was removed after 2 weeks. t: a modified e-PTFE membrane was used in this group. §: For this study were reported baseline and follow-up data but not the differences. $\int$ : At 12 months author reported only data on pocket closure. bTCP: Tricalcium Phosphate; CAF: Coronally advanced flap; DFDBA: demineralized freeze-dried bone allograft; EMD: enamel matrix derivative; GTR-NR: guided tissue regeneration using a non-resorbable membrane; GTR-R: GTR using a resorbable membrane; HA: hydroxyapatite; OFD: open flap debridement. 


\subsection{Risk of Bias}

Out of the 105 included studies, 17 were rated at high risk of bias, 68 at unclear and only 20 at low risk of bias. Among the seven domains, the lack of blinding of the outcome assessor and incomplete outcome data were the most frequent sources of bias for both ID and FI studies (Supplementary Material S5).

\subsection{Pair-Wise Meta-Analyses}

Intrabony defects: A total of four included studies tested adjunctive antibiotics in regenerative therapy of ID [11,18-20]. Among these, three could be included in metaanalysis [18-20], as the other did not report standard deviations. One of these studies used EMD + BRG (with or without amoxicillin) [18], the second EMD (with or without Doxycycline) ([19], and the third used GTR-R + BRG (with or without amoxicillin) ([20]. Pair-wise meta-analyses (Figure 1) showed lack-of statistically significant differences for antibiotic vs. no-antibiotic for PPD reduction $\left(-0.43 \mathrm{~mm}, 95 \% \mathrm{CI}=-1.20 ; 0.33, \mathrm{I}^{2}=0 \%\right)$, CAL gain $\left(-0.27 \mathrm{~mm}, 95 \% \mathrm{CI}=-0.90 ; 0.36, \mathrm{I}^{2}=28 \%\right)$ and bone gain $(-0.35 \mathrm{~mm}, 95 \% \mathrm{CI}=-1.31$; $\left.0.61, \mathrm{I}^{2}=0 \%\right)$.

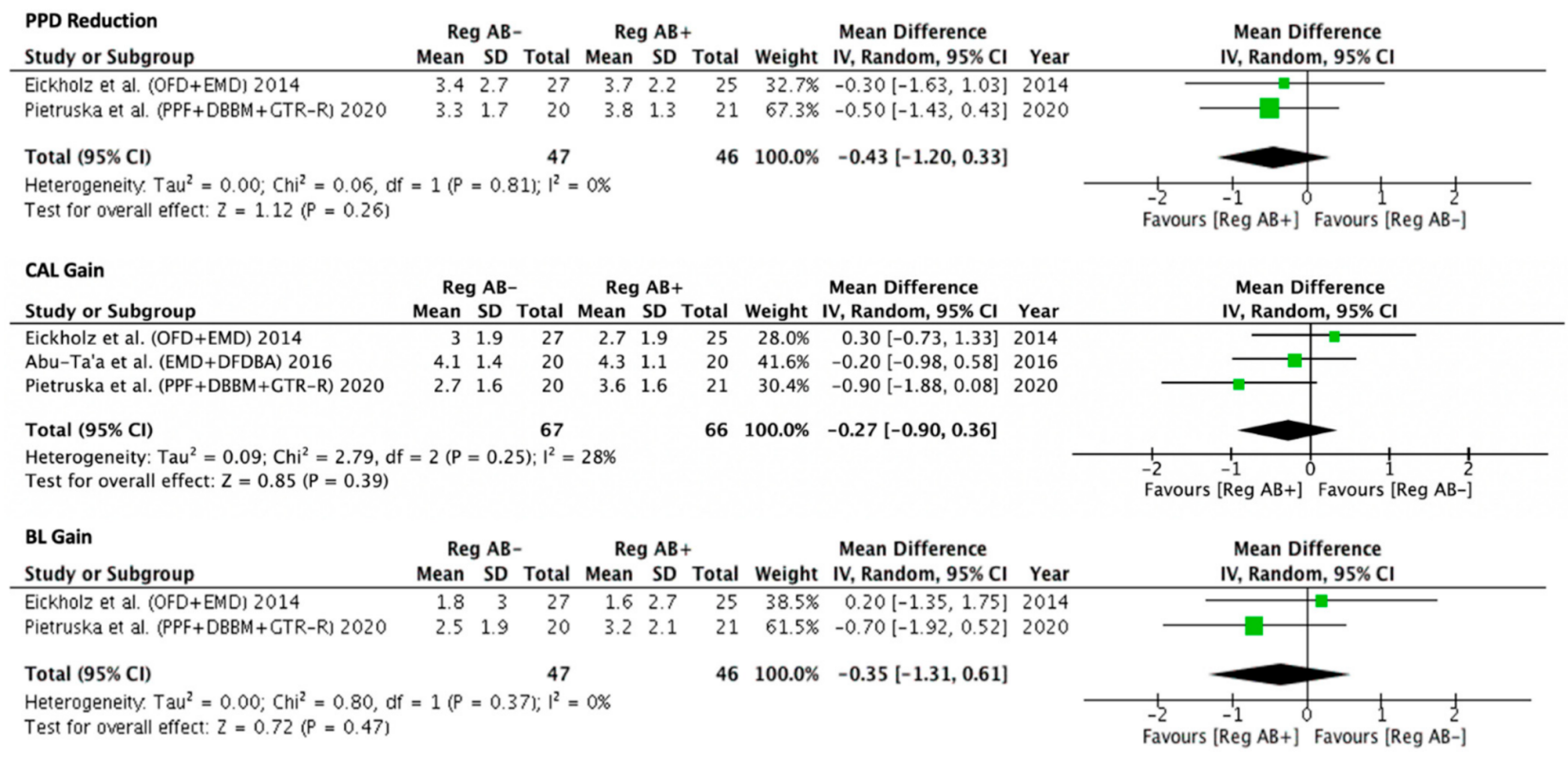

Figure 1. Forest plots of pair-wise meta-analyses for PPD reduction, CAL gain and bone gain for studies directly comparing regenerative techniques in intrabony defects with or without adjunctive antibiotics.

Furcation involvement: No studies were available for pair-wise meta-analyses of $\mathrm{AB}$ vs. non- $\mathrm{AB}$ for FI.

\subsection{Single-Arm Meta-Regression for Intrabony Defects}

Single-arm meta-analyses for intrabony defects included between 39 and 82 antibiotic arms and between 15 and 32 non-antibiotic arms (depending on outcomes reported). Studies included EMD, GTR with resorbable and non-resorbable membranes, with or without bone replacement grafts. Antibiotics employed included penicillin, amoxicillin, amoxicillin + clavulanic acid, ampicillin, doxycycline, tetracycline, cephalosporin and clindamycin. The following outcomes were statistically significantly improved in AB vs. non- $\mathrm{AB}$ study arms (see summary in Table 3 and Forest Plots in Supplementary Material S6): 
Table 3. Summary of meta-regressions for studies on ID.

\begin{tabular}{|c|c|c|c|c|c|}
\hline Treatment & Number of Arms & Outcome & Difference & CI & $p$-Value \\
\hline \multirow{3}{*}{ Overall/any treatment } & $\begin{array}{l}\mathrm{AB}-(32 \mathrm{arms}) \\
\mathrm{AB}+(82 \mathrm{arms})\end{array}$ & PPD & $-0.91 \mathrm{~mm}$ & -1.3 to -0.51 & $<0.001$ \\
\hline & $\begin{array}{l}\mathrm{AB}-(32 \mathrm{arms}) \\
\mathrm{AB}+(82 \mathrm{arms})\end{array}$ & CAL & $-0.92 \mathrm{~mm}$ & -1.32 to -0.52 & $<0.001$ \\
\hline & $\begin{array}{l}\mathrm{AB}-(15 \mathrm{arms}) \\
\mathrm{AB}+(39 \mathrm{arms})\end{array}$ & BG & $-1.1 \mathrm{~mm}$ & -1.63 to -0.53 & $<0.001$ \\
\hline \multirow{2}{*}{ EMD } & $\begin{array}{l}\mathrm{AB}-(13 \mathrm{arms}) \\
\mathrm{AB}+(15 \mathrm{arms})\end{array}$ & PPD & $-0.98 \mathrm{~mm}$ & -1.65 to -0.32 & 0.004 \\
\hline & $\begin{array}{l}\mathrm{AB}-(13 \mathrm{arms}) \\
\mathrm{AB}+(15 \mathrm{arms})\end{array}$ & CAL & $-0.98 \mathrm{~mm}$ & -1.77 to -0.19 & 0.015 \\
\hline \multirow{2}{*}{ BRG + GTR-R } & $\begin{array}{l}A B-(10 \text { arms }) \\
A B+(23 \text { arms })\end{array}$ & PPD & $-0.22 \mathrm{~mm}$ & -0.94 to 0.5 & 0.553 \\
\hline & $\begin{array}{l}\mathrm{AB}-(10 \mathrm{arms}) \\
\mathrm{AB}+(23 \mathrm{arms})\end{array}$ & CAL & $-0.15 \mathrm{~mm}$ & -0.91 to 0.6 & 0.688 \\
\hline \multirow{2}{*}{ Type of AB } & $\begin{array}{l}\text { Tetracycline (17 arms) } \\
\text { Penicillin (55 arms) }\end{array}$ & PPD & $-0.25 \mathrm{~mm}$ & -0.79 to 0.29 & 0.360 \\
\hline & $\begin{array}{l}\text { Tetracycline (17 arms) } \\
\text { Penicillin (55 arms) }\end{array}$ & CAL & $-0.24 \mathrm{~mm}$ & -0.73 to 0.25 & 0.338 \\
\hline
\end{tabular}

$\mathrm{AB}-$ : no antibiotic; $\mathrm{AB}+$ : antibiotic; $\mathrm{PPD}$ : probing pocket depth; $\mathrm{CAL}$ : clinical attachment level; $\mathrm{BG}$ : bone gain; EMD: enamel matrix derivative; BRG: bone replacement graft; GTR-R: guided tissue regeneration by mean resorbable membrane.

PPD reduction: $4.56 \mathrm{~mm}\left(95 \% \mathrm{CI}=4.33 ; 4.79, \mathrm{I}^{2}=95.73 \%\right)$ in $\mathrm{AB}$ arms vs. $3.64 \mathrm{~mm}$ $\left(95 \% \mathrm{CI}=3.35 ; 3.94 \mathrm{~mm}, \mathrm{I}^{2}=88.98 \%\right)$ in non $-\mathrm{AB}$ arms (meta regression mean diff: -0.91 , $95 \% \mathrm{CI}=-1.30 ;-0.51, p<0.001)$.

CAL gain: $3.71 \mathrm{~mm}\left(95 \% \mathrm{CI}=3.49 ; 3.94, \mathrm{I}^{2}=96.05 \%\right)$ in $\mathrm{AB}$ arms vs. $2.79 \mathrm{~mm}(95 \% \mathrm{CI}=2.33$; $3.26 \mathrm{~mm}, \mathrm{I}^{2}=96.78 \%$ ) in non $-\mathrm{AB}$ arms (meta regression mean diff: $-0.92,95 \% \mathrm{CI}=-1.32$; $-0.52, p<0.001)$.

Bone gain: $3.26 \mathrm{~mm}\left(95 \% \mathrm{CI}=2.99 ; 3.53, \mathrm{I}^{2}=93.71 \%\right)$ in $\mathrm{AB}$ arms vs. $2.17 \mathrm{~mm}$ $\left(95 \% \mathrm{CI}=1.79 ; 2.56 \mathrm{~mm}, \mathrm{I}^{2}=85.06 \%\right)$ in non $-\mathrm{AB}$ arms (meta-regression mean diff: -1.08 , $95 \% \mathrm{CI}=-1.63 ;-0.53, p<0.001)$.

Sub-analyses (meta-regression) by biomaterial in intrabony defects showed better clinical outcomes in studies using EMD in AB arms vs. non-AB arms for PPD reduction (meta-regression mean diff: $-0.98,95 \% \mathrm{CI}=-1.65 ;-0.32, p=0.004$ ) and for CAL gain (meta-regression mean diff: $-0.98,95 \% \mathrm{CI}=-1.77 ;-0.19, p=0.015$ ) (Table 3 and Supplementary Material S7); however, no statistically significant differences were estimated between $\mathrm{AB}$ and non- $\mathrm{AB}$ arms in studies using bone grafts and GTR with resorbable membranes for PPD reduction (meta-regression mean diff: $-0.22,95 \% \mathrm{CI}=-0.94 ; 0.50$, $p=0.553$ ) and for CAL gain (meta-regression mean diff: $-0.15,95 \% \mathrm{CI}=-0.91 ; 0.60$, $p=0.688$ ) (Table 3 and Supplementary Material S8).

Sub-analyses (meta-regression) by antibiotic type in intrabony defects showed no statistically significant differences in studies using antibiotics of the penicillin family (including $55 \mathrm{arms}$ ) vs. studies using tetracyclines (including $17 \mathrm{arms}$ ) for PPD reduction (meta-regression mean diff: $-0.25,95 \% \mathrm{CI}=-0.79 ; 0.29, p=0.360$ ) and for CAL gain (metaregression mean diff: $-0.24,95 \% \mathrm{CI}=-0.73 ; 0.25, p=0.338$ ) (Table 3 and Supplementary Material S9).

\subsection{Single-Arm Meta-Regression for Furcation Defects}

Single-arm meta-analyses for furcation defects included between 9 and 21 antibiotic arms and between 2 and 7 non-antibiotic arms (depending on outcomes reported). Studies included a combination of EMD, GTR with resorbable and non-resorbable membranes and 
bone replacement grafts, alone or in combination. Antibiotics employed included penicillin, amoxicillin, amoxicillin + clavulanic acid, doxycycline and tetracycline. Non-statistically significant differences between $\mathrm{AB}$ and non- $\mathrm{AB}$ groups were estimated for:

PPD reduction: $2.03 \mathrm{~mm}\left(95 \% \mathrm{CI}=1.76 ; 2.31, \mathrm{I}^{2}=93.92 \%\right)$ in $\mathrm{AB}$ arms vs. $2.26 \mathrm{~mm}$ $\left(95 \% \mathrm{CI}=2.05 ; 2.47, \mathrm{I}^{2}=17.04 \%\right)$ in non- $\mathrm{AB}$ arms (meta-regression mean diff: $0.27,95 \% \mathrm{CI}=-0.45$; $0.98, p=0.465)$ (Figure 2).

Studies

Blumenthal et al. (OFD+GTR-R) 1993 Bouchard et al. (OFD+GTR-R) 1993

Pruthi et al. (OFD+GTR-R) 2002 Wang et al. (OFD+GTR-R) 1994

de Leonardis et al. (OFD+GTR-R) 1999 Garrett et al. (OFD+GTR-R) 1997

Bouchard et al. (OFD+GTR-R) 1997

Blumenthal et al. (OFD+GTR-NR) 1993

Bouchard et al. (OFD+GTR-NR) 1993

Pruthi et al. (OFD+GTR-NR) 2002

Garrett et al. (OFD+GTR-NR) 1997

Bouchard et al. (OFD+GTR-NR) 1997

Leite et al. (OFD+GTR-NR) 2013a

Leite et al. (OFD+GTR-NR) 2013b

Garrett et al. (OFD+DFDBA) 1990

Garrett et al. (CAF+GTR-R+DFDBA) 1990

Jaiswal et al. (OFD+GTR-R+DFDBA) 2013

de Leonardis et al. (OFD+GTR-R+DFDBA) 199 Jaiswal et al. (OFD+GTR-R+DFDBA+EMD) 2013

Santana et al. (CAF+GTR-NR+HA) 2009

de Santana et al. (CAF+GTR-R+HA) 1999

Subgroup $A B+\left(I^{\wedge} 2=9392 \%, P=0.000\right)$

Hugoson et al. (OFD+GTR-R) 1995 Hugoson et al. (OFD+GTR-NR) 1995 Queiroz et al. (OFD+EMD) 2016 Queiroz et al. (OFD+bTCP/HA) 2016 Queiroz et al. (OFD+bTCP/HA+EMD) 2016

Subgroup AB- $\left(\left.\right|^{\wedge} 2=1704 \%, P=0.306\right)$

Overall $\left(\left.\right|^{\wedge} 2=92.51 \%, P=0.000\right)$

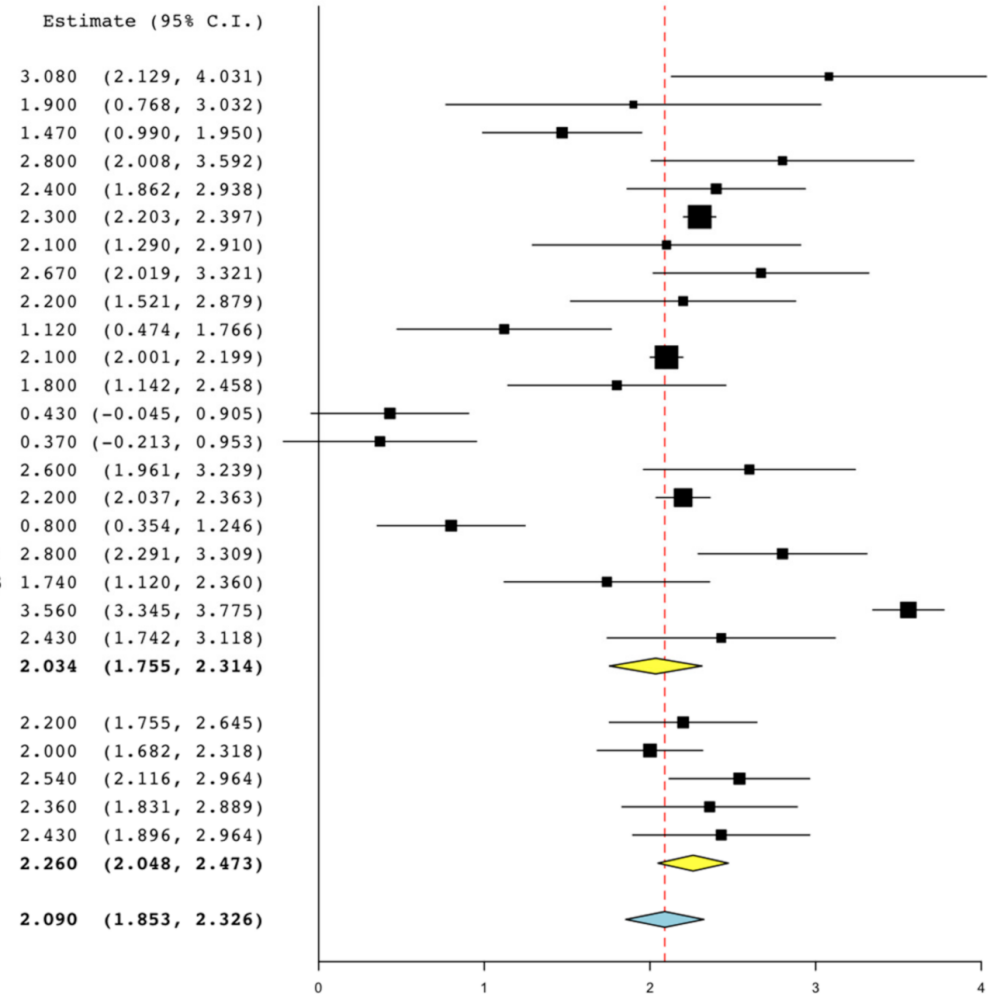

Figure 2. Forest plots of single-arm meta-analyses for PPD reduction for studies of regenerative techniques in furcation defects with or without adjunctive antibiotics.

H-BL gain: $1.56 \mathrm{~mm}\left(95 \% \mathrm{CI}=0.99 ; 2.13, \mathrm{I}^{2}=93.99 \%\right)$ in $\mathrm{AB}$ arms vs. $2.23 \mathrm{~mm}(95 \% \mathrm{CI}=1.55$; $2.92, \mathrm{I}^{2}=76.42 \%$ ) in non-AB arms (meta-regression mean diff: $0.69,95 \% \mathrm{CI}=-0.39 ; 1.78$, $p=0.209$ ) (Figure 3).

Studies

Blumenthal et al. (OFD+GTR-R) 1993 Bouchard et al. (OFD+GTR-R) 1993

Pruthi et al. (OFD+GTR-R) 2002

Blumenthal et al. (OFD+GTR-NR) 1993 Bouchard et al. (OFD+GTR-NR) 1993

Pruthi et al. (OFD+GTR-NR) 2002 Leite et al. (OFD+GTR-NR) 2013

Leite et al. (OFD+GTR-NR) 2013b

Garrett et al. (OFD+DFDBA) 1990

Garrett et al. (CAF+GTR-R+DFDBA) 19

Subgroup $A B+\left(I^{\wedge} 2=9299 \%, P=0.000\right)$

Jepsen et al. (OFD+EMD) 2004 Jepsen et al. (OFD+GTR-R) 2004

Subgroup $A B-\left(I^{\wedge} 2=7642 \%, P=0.039\right)$

Overall ( $\left(\left.\right|^{\wedge} 2=92.93 \%, P=0.000\right)$
Estimate (958 C.I.)

$2.500(2.104,2.896)$

$1.500(0.878,2.122)$

$0.410(0.072,0.748)$

$1.700(1.417,1.983)$

$2.200(1.408,2.992)$

$0.410(0.115,0.705)$

$1.360(0.472,2.248)$

$1.180(0.569,1.791)$

$2.600(1.978,3.222)$

$2.000(0.858,3.142)$

$1.560(0.992,2.128)$

$2.600(2.074,3.126)$

$1.900(1.491,2.309)$

$2.230(1.545,2.915)$

$1.679(1.168,2.190)$

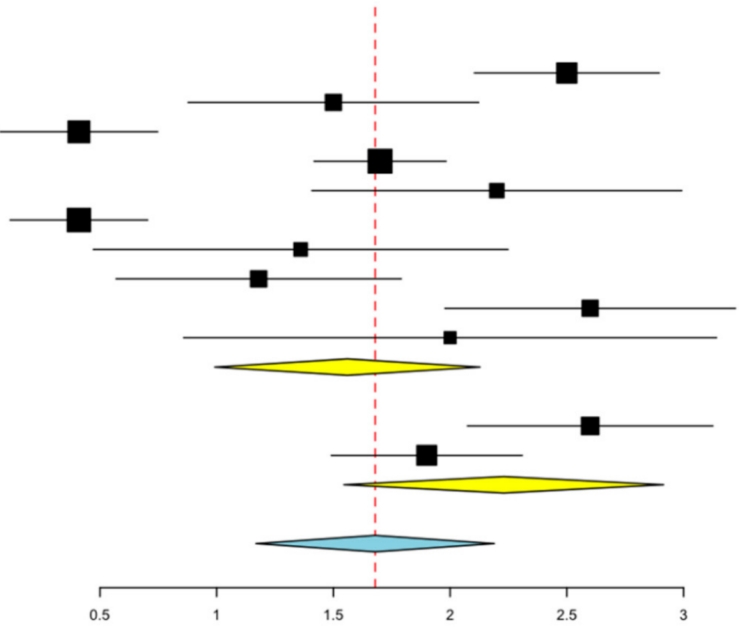

Figure 3. Forest plots of single-arm meta-analyses for H-BL gain for studies of regenerative techniques in furcation defects with or without adjunctive antibiotics. 
V-CAL gain: $1.50 \mathrm{~mm}\left(95 \% \mathrm{CI}=1.26 ; 1.72, \mathrm{I}^{2}=96.16 \%\right)$ in $\mathrm{AB}$ arms vs. $1.30 \mathrm{~mm}$ $\left(95 \% \mathrm{CI}=0.70 ; 1.90, \mathrm{I}^{2}=84.59 \%\right)$ in non-AB arms (meta-regression mean diff: -0.16 , $95 \% \mathrm{CI}=0.81 ; 0.50, p=0.638$ ) (Figure 4 ).

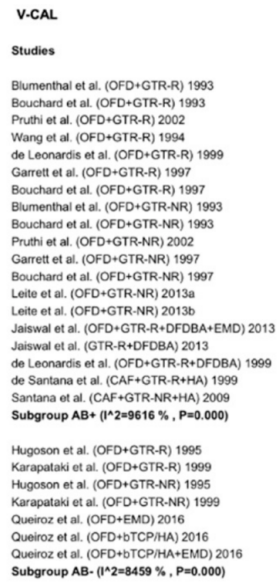

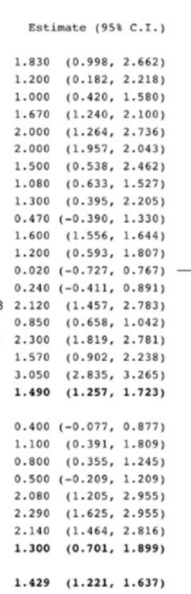
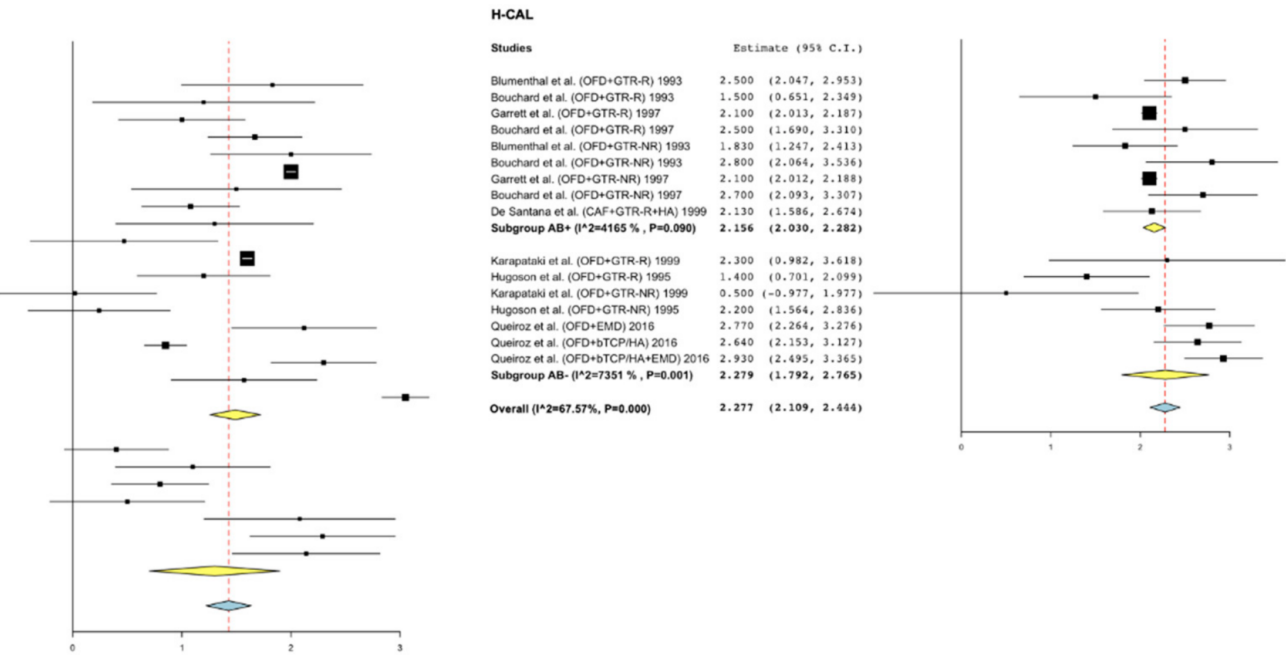

Figure 4. Forest plots of single-arm meta-analyses for V-CAL and H-CAL gain for studies of regenerative techniques in furcation defects with or without adjunctive antibiotics.

H-CAL gain: $2.16 \mathrm{~mm}\left(95 \% \mathrm{CI}=2.03 ; 2.28 \mathrm{~mm}, \mathrm{I}^{2}=41.65 \%\right)$ in AB arms vs. $2.28 \mathrm{~mm}$ $\left(95 \% \mathrm{CI}=1.79 ; 2.77 \mathrm{~mm}, \mathrm{I}^{2}=73.51 \%\right)$ in non-AB arms (meta-regression mean diff: 0.16 , $95 \% \mathrm{CI}=0.26 ; 0.57, p=0.452$ ) (Figure 4 ).

Sub-analysis by antibiotic type in furcation defects showed no statistically significant differences in studies using antibiotics of the penicillin family and studies using tetracyclines for PPD reduction (meta-regression mean diff: $0.34,95 \% \mathrm{CI}=0.36 ; 1.05, p=0.342$ ) and for CAL gain (meta-regression mean diff: $0.31,95 \% \mathrm{CI}=-0.34 ; 0.96, p=0.346$ ) (Table 3 and Supplementary Material S10).

\subsection{Adverse Events/Post-Operative Infections}

No serious adverse events were reported in the studies included in this review.

\subsubsection{Pair-Wise Meta-Analysis Studies (ID)}

Regarding the three studies included in the pair-wise meta-analysis of ID, Abu ta'a et al. [18] who had used EMD + BRG, stated that apart from erythema and swelling, no more severe postoperative complications such as suppuration, sloughing, perforation of the flap, and postoperative pain were reported in either of the groups. No information on adverse events / post-operative infection was provided by Eickholz et al. [19] who had employed EMD, whereas Pietruska et al. [20] that had used GTR-R + BRG reported that no signs of suppuration, extensive dehiscence or swelling were observed throughout the entire study period and that none of the patients reported intense pain, fever or discomfort.

\subsubsection{Single-Arm Meta-Regression Studies (ID)}

Sixty-nine studies (55 AB+, $14 \mathrm{AB}-$ ) included in the single-arm meta-regression for ID clearly reported that there were no adverse events or post-operative infections during the healing period. In four studies (all $\mathrm{AB}+$ ), no information on absence or presence of adverse events/post-operative infection was provided [21-24]. Another eight studies (AB+ and $\mathrm{AB}-$ ) reported on unwanted postoperative healing events such as membrane exposure, slight inflammation, pain, swelling and bleeding but did not label these as adverse events or post-operative infections [25-32]. Only four ID studies specifically reported adverse events and/or postoperative infections. In the $\mathrm{AB}-$ arm of one study [11], a post-operative abscess occurred following the use of a resorbable membrane in one patient and was treated 
with antibiotics. Another infection of a site treated with a non-resorbable membrane was reported in a patient $(A B+)$ after the surgery [33]. A pulp necrosis was observed in a study $(\mathrm{AB}+)$ using EMD [34]. In another study $(\mathrm{AB}-)$ using non-resorbable titanium-reinforced membranes, three patients were treated with antibiotics after membrane exposure without infection or abscesses [35]. In summary, adverse events/post-operative infections after regenerative treatment of IDs were rare.

\subsubsection{Single-Arm Meta-Regression Studies (FI)}

For FI, in six of the studies ( $5 \mathrm{AB}+, 1 \mathrm{AB}-)$ included in the single-arm meta-regression, no information on post-operative adverse events or infections was reported at all. An additional seven studies (all $\mathrm{AB}+$ ) explicitly stated that no post-operative infections or adverse tissue reactions had occurred. The remaining seven studies $(4 \mathrm{AB}+, 3 \mathrm{AB}-)$ listed a few adverse healing events. Two studies $(A B+)$ observed one abscess each following the use of a non-resorbable membrane [36,37]. Another parallel group study $(A B+)$ reported 4 (out of 66) molars with abscess formation in the GTR-R group and 10 (out of 64) abscesses in the GTR-NR group [38]. In a split-mouth study $(\mathrm{AB}-$ ), 13 (out of 38) patients had to receive antibiotics due to postoperative swelling, pain and suppuration, more frequently associated with the teeth that had been treated with GTR-NR [39]. In another split-mouth study $(\mathrm{AB}-$ ) in 4 (out of 11) patients, antibiotics were prescribed because of postoperative pain, swelling and suppuration following GTR-R and GTR-NR [40]. In the third (AB-) study [41], antibiotics were given due to signs of post-operative infection in 8 (out of 45) teeth following GTR-R and in 2 (out of 45) teeth following EMD treatment.

In summary, post-operative infections after regenerative treatment of FI occurred infrequently in studies and treatment arms with and without a post-operative antibiotic protocol and appeared to be mostly related to the use of non-resorbable membranes.

The exposure of the membrane, biomaterial and the lack of primary closure were the most common complications reported in both studies with and without antibiotic treatment. The incidence, the assessment and the influence of these complications on the regenerative outcomes vary across the studies; thus, a meta-analysis was not considered appropriate.

\section{Discussion}

This systematic review provides weak evidence that adjunctive systemic antibiotics could lead to slight additional clinical and radiographic benefits as part of the surgical regenerative periodontal treatment of intrabony defects. No such evidence could be obtained with regard to the regenerative treatment of furcation defects.

This systematic review addresses a very important question. The use of antibiotics in periodontology is still controversial. Studies have shown that the development of antibiotic resistance in periodontal pathogens exists [42-44]. A high proportion of patients with resistant pathogens was described by Rams et al. [42], where $74 \%$ of patients were reported to have subgingival periodontal pathogens resistant to at least one of the antibiotics commonly used in clinical practice. Recently, periodontal pathogens collected from almost 8000 patients with periodontitis were shown to be non-susceptible to at least one of the antibiotics tested in about two-thirds of the patients [44] and the data further revealed a trend towards decreasing susceptibility profiles. While a targeted antimicrobial approach, based on microbial analysis, may be considered in the context of step 2 of periodontal therapy [6], aiming at the reduction in the causative agents (subgingival bacterial biofilm on the root surface), this may not be appropriate for step 3 therapy, where the aim of adjunctive antibiotics is prevention/prophylaxis of wound/biomaterial infection. In the era of increasing antimicrobial resistance, public health needs demand a reduction in the use of unnecessary antibiotic usage through antibiotic stewardship [45]. Although adjunctive systemic antibiotics have been shown to lead to enhanced clinical and radiographic outcomes following non-surgical periodontal therapy [3], a recent S3-level clinical guideline calls for a rather restrictive use in the non-surgical phase [6]; however, no evidence-based guidelines deal with the adjunctive prophylactic use of antibiotics in conjunction with periodontal 
surgery $[12,13,46,47]$. Despite their common use in practice, it has been unclear if their use in this scenario is justifiable. The data produced by this systematic review can contribute to the debate about whether systemic antibiotics should be prescribed concomitantly to regenerative periodontal surgery.

Traditional meta-analysis could only be performed for data derived by two RCTs in ID and revealed the absence of adjunctive clinical benefits associated with the use of systemic AB. This is in agreement with some other studies on this topic, which could not be included in the meta-analysis due to the review's inclusion criteria or lack of reported data $[10,11]$; however, meta-regression of single-arm studies showed a higher magnitude of improvements of most investigated clinical and radiographic measures in ID. In particular, PPD and CAL were improved more in 'antibiotic' arms compared with 'non-antibiotic arms', both with differences just short of $1 \mathrm{~mm}$. The radiographic bone gain was just over $1 \mathrm{~mm}$ higher in 'antibiotic arms' compared with 'non-antibiotic arms'. It could be speculated that this difference may be due to the reduced risk of infection during the healing process, which may impair the regenerative process, especially at early stages. Although the potential benefit of prescribing systemic antibiotics is often sought when applying barrier membranes and/or bone substitutes, the data analyzed in this review pointed towards an increased benefit in studies using EMD, rather than in studies using GTR with resorbable membranes and bone grafts. Even if modern literature has extensively demonstrated that papillary preservation flaps are mandatory for effective regenerative procedures [7], systemic antibiotics may increase the protection of regeneration sites against infection sources in the oral cavity in conjunction with primary wound closure. In this context, it is interesting that the additional clinical and radiographic improvements appeared only in intrabony studies but not for regenerative/reconstructive surgery of furcation defects. It may be speculated that this could be due to the challenge of primary flap closure for IDs, mostly located interdentally. In contrast, flap access and adequate wound closure are less demanding in sites with FI, in studies mostly located buccally. It should be added that although adjunctive antibiotic therapy may be recommended in immunocompromised patients or individuals with high infection risk, such patients are usually excluded from studies on regenerative periodontal treatment, and often from periodontal surgery in clinical practice.

No serious adverse events were reported in any of the included studies. Thus, regenerative treatments at both ID and FI, with or without systemic antibiotics, could be considered safe procedures. In 86 studies on ID, only two patients with post-operative infections, one in an $\mathrm{AB}+$ and another in an $\mathrm{AB}$ - group, were reported. This is in contrast to the 19 studies on FI, where 16 out of 157 patients $(10 \%)$ in $\mathrm{AB}+$ study arms of four studies experienced post-operative abscesses, whereas 25 out of 139 patients $(18 \%)$ in $\mathrm{AB}-$ study arms (three studies) experienced postoperative pain, swelling and suppuration, that were treated by antibiotics. Thus, very little data are available on post-operative infections. Moreover, they occurred in study arms with and without post-operative administration of systemic antibiotics and, therefore, it is difficult to assess the influence of the use of AB. Interestingly, almost all the infections occurred in sites with a barrier membrane. In one RCT, $100 \%$ of the defects treated with a resorbable membrane showed at least a complication compared to 6\% of EMD-treated defects [29]. Barrier membrane exposure was the most common complication reported in the studies included in this review. These data support the hypothesis that the use of barrier membranes could increase the risk of infection during the early healing stages. In this context, the protective effects of adjunctive $A B$ on membrane exposure remain unclear. A recent RCT tested the effect of an $A B$ on regeneration by means of DBBM and a resorbable membrane. Membrane exposure was reported in seven control patients not receiving $\mathrm{AB}$ and six test patients receiving $\mathrm{AB}$. No signs of swelling or suppuration were reported in any case [20]. Interestingly, another randomized controlled trial showed that patients' subjective perception of postoperative discomfort was significantly smaller in the group receiving antibiotics compared with the placebo group [18]. 
Sub-analysis on the type of adjunctive antibiotics used showed no differences in PD and CAL gain in ID when antibiotics of the penicillin or tetracycline families were used. This needs to be interpreted cautiously due to the lack of direct comparisons.

The strength of this review is that it is the first, to our knowledge, to combine data on regenerative therapy of intrabony defects and furcations to assess the potential effect of adjunctive antibiotics. Furthermore, a very large number of studies were included. Limitations include high heterogeneity of most of the analyses and the fact that less than $20 \%$ of included papers were judged to have a low risk of bias, with items such as lack of blinding of the outcome assessor and incomplete outcome data considered as the most frequent sources of bias. The heterogeneity is likely due to the high variability in regenerative procedures and antibiotic protocols among selected trials. This heterogeneity may hinder the final selection of the proper approach in infection prevention. It should also be stressed that, due to the nature of the meta-regression, we cannot conclude that the observed differences between antibiotic and non-antibiotic arms were entirely due to the use of antibiotics.

Thus, future research in this area may be necessary by well-designed RCTs to prove or disprove the benefits of adjunctive systemic antibiotics during regenerative/reconstructive surgery. It will be important to standardize protocols treatment protocols, for example, by including in future RCTs testing adjunctive systemic antibiotics in step 3 of periodontal therapy only patients who received or did not receive such antibiotics in step 2.

However, recent reviews and surveys have emphasized the low incidence of infection in periodontal surgery irrespective of the use of systemic antibiotics, have suggested not to use them in the perioperative period to prevent infection [12] and have demanded the development of guidelines [13,47].

\section{Conclusions}

At present, based on the results of the present systematic review, with the available weak evidence for additional benefits attributable to systemic antibiotics in the regenerative/reconstructive periodontal surgery of intrabony defects and no evidence for their additional benefit in furcation defects, it appears questionable to justify their adjunctive use.

Supplementary Materials: The following are available online at https:/ /www.mdpi.com/article / 10.3390/antibiotics11010008/s1, Material S1: PRISMA checklist; Material S2: Search strategy and study selection; Material S3: Full list of included papers; Material S4: Flow Chart diagram (modified from PRISMA); Material S5: Risk of bias; Material S6: Forest plots of single-arm meta-analyses and meta-regression models using $\mathrm{AB}$ as factor in intrabony defects; Material S7: Meta-regression using $\mathrm{AB}$ as factor in intrabony defects for the EMD subgroup; Material S8: Meta-regression using $\mathrm{AB}$ as factor in intrabony defects for the Graft + / - GTR-R subgroup; Material S9: Meta-regression using $\mathrm{AB}$ type as factor in intrabony defects; Material S10: Meta-regression using AB type as factor in furcation defects.

Author Contributions: Conceptualization, Manuscript writing L.N. and S.J.; Data curation, L.B.; Formal analysis, J.B.; Investigation, F.C. and F.G. All authors have read and agreed to the published version of the manuscript.

Funding: This research received no external funding.

Conflicts of Interest: The authors have stated explicitly that there are no conflicts of interest in connection with this article.

\section{References}

1. Baer, P.N.; Socransky, S.S. Periodontosis: Case report with long-term follow-up. Periodontal Case Rep. Publ. Northeast. Soc. Periodontists 1979, 1, 1-6.

2. van Winkelhoff, A.J.; Rodenburg, J.P.; Goene, R.J.; Abbas, F.; Winkel, E.G.; de Graaff, J. Metronidazole plus amoxycillin in the treatment of Actinobacillus associated periodontitis. J. Clin. Periodontol. 1989, 16, 128-131. [CrossRef] [PubMed]

3. Teughels, W.; Feres, M.; Oud, V.; Martín, C.; Matesanz, P.; Herrera, D. Adjunctive ef-fect of systemic antimicrobials in periodontitis therapy: A systematic review and meta-analysis. J. Clin. Periodontol. 2020, 47, 257-281. [CrossRef] [PubMed] 
4. Jepsen, K.; Jepsen, S. Antibiotics/antimicrobials: Systemic and local administration in the therapy of mild to moderately advanced periodontitis. J. Periodontol. 2000 2016, 71, 82-112. [CrossRef]

5. WHO Global Antimicrobial Resistance Surveillance System (GLASS) Report: Early Implementation 2017-2018; WHO: Geneva, Switzerland, 2018.

6. Sanz, M.; Herrera, D.; Kebschull, M.; Chapple, I.; Jepsen, S.; Berglundh, T.; Sculean, A.; Tonetti, M.S.; Aass, A.M.; Aimetti, M.; et al. Treatment of stage I-III periodontitis-The EFP S3 level clinical practice guideline. J. Clin. Periodontol. 2020, 47, 4-60. [CrossRef]

7. Nibali, L.; Koidou, V.P.; Nieri, M.; Barbato, L.; Pagliaro, U.; Cairo, F. Regenera-tive surgery versus access flap for the treatment of intraos- seous periodontal defects. A systematic review and meta-analysis. J. Clin. Periodontol. 2020, 47, 320-351. [CrossRef] [PubMed]

8. Jepsen, S.; Gennai, S.; Hirschfeld, J.; Kalemaj, Z.; Buti, J.; Graziani, F. Regenerative surgical treatment of furcation defects: A systematic review and Bayesian network meta-analysis of randomized clinical trials. J. Clin. Periodontol. 2020, 47, 352-374. [CrossRef]

9. Sculean, A.; Blaes, A.; Arweiler, N.; Reich, E.; Donos, N.; Brecx, M. The effect of postsurgical antibiotics on the healing of intrabony defects following treat-ment with enamel matrix proteins. J. Periodontol. 2001, 72, 190-195. [CrossRef] [PubMed]

10. Minabe, M.; Kodama, T.; Kogou, T.; Fushimi, H.; Sugiyama, T.; Takeuchi, K.; Miterai, E.; Nishikubo, S. Clinical significance of antibiotic therapy in guided tissue regenera-tion with a resorbable membrane. Periodontal Clin. Investig. 2001, 23, 20-30.

11. Loos, B.G.; Louwerse, P.H.G.; Van Winkelhoff, A.J.; Burger, W.; Gilijamse, M.; Hart, A.A.M.; Van Der Velden, U. Use of barrier membranes and systemic antibiotics in the treatment of intraosseous defects. J. Clin. Periodontol. 2002, 29, 910-921. [CrossRef]

12. Liu, Y.; Duan, D.; Xin, Y.; Bai, L.; Li, T.; Li, C.; Xu, Y. A review of the literature: Antibiotic usage and its relevance to the infection in periodontal flaps. Acta Odontol. Scand. 2017, 75, 288-293. [CrossRef]

13. Hai, J.H.; Lee, C.; Kapila, Y.L.; Chaffee, B.W.; Armitage, G.C. Antibiotic prescribing practices in periodontal surgeries with and without bone grafting. J. Periodontol. 2020, 91, 508-515. [CrossRef]

14. Moher, D.; Liberati, A.; Tetzlaff, J.; Altman, D.G. Preferred Reporting Items for Systematic Reviews and Meta-Analyses: The PRISMA Statement. J. Clin. Epidemiol. 2009, 62, 1006-1012. [CrossRef]

15. Moher, D.; Shamseer, L.; Clarke, M.; Ghersi, D.; Liberati, A.; Petticrew, M.; Shekelle, P.; Stewart, L.A.; Group, P.-P. Preferred reporting items for systematic review and meta-analysis protocols (PRISMA-P) 2015 statement. Syst. Rev. 2015, 4, 1. [CrossRef]

16. Page, M.J.; McKenzie, J.E.; Bossuyt, P.M.; Boutron, I.; Hoffmann, T.C.; Mulrow, C.D.; Shamseer, L.; Tetzlaff, J.M.; Akl, E.A.; Brennan, S.E.; et al. The PRISMA 2020 statement: An updated guideline for reporting systematic reviews. BMJ 2021, $372, \mathrm{n} 71$. [CrossRef]

17. Higgins, J.P.; Green, S. (Eds.) Cochrane Handbook for Systematic; Reviews of Interventions Version 5.1.0.; The Cochrane Collaboration: London, UK, 2011; Available online: www.cochrane-handbook.org (accessed on 1 July 2021).

18. Abu-Ta'A, M. Adjunctive Systemic Antimicrobial Therapy vs. Asepsis in Conjunction with Guided Tissue Regeneration: A Randomized, Controlled Clinical Trial. J. Contemp. Dent. Pract. 2016, 17, 3-6. [CrossRef]

19. Eickholz, P.; Röllke, L.; Schacher, B.; Wohlfeil, M.; Dannewitz, B.; Kaltschmitt, J.; Krieger, J.K.; Krigar, D.M.; Reitmeir, P.; Kim, T.-S. Enamel Matrix Derivative in Propylene Glycol Alginate for Treatment of Infrabony Defects with or Without Systemic Doxycycline: 12- and 24-Month Results. J. Periodontol. 2014, 85, 669-675. [CrossRef]

20. Pietruska, M.; Dolińska, E.; Milewski, R.; Sculean, A. Effect of systemic antibiotics on the outcomes of regenerative periodontal surgery in intrabony defects: A randomized, controlled, clinical study. Clin. Oral Investig. 2021, 25, 2959-2968. [CrossRef]

21. Silvestri, M.; Ricci, G.; Rasperini, G.; Sartori, S.; Cattaneo, V. Comparison of treatments of infrabony defects with enamel matrix derivative, guided tissue regeneration with a nonresorbable membrane and Widman modified flap. A pilot study. J. Clin. Periodontol. 2000, 27, 603-610. [CrossRef]

22. Silvestri, M.; Sartori, S.; Rasperini, G.; Ricci, G.; Rota, C.; Cattaneo, V. Comparison of infrabony defects treated with enamel matrix derivative versus guided tissue regeneration with a nonresorbable membrane. J. Clin. Periodontol. 2003, 30, 386-393. [CrossRef]

23. Rösing, C.K.; Aass, A.M.; Mavropoulos, A.; Gjermo, P. Clinical and radiographic effects of enamel matrix derivative in the treatment of intrabony periodontal defects: A 12-month longitudinal placebo-controlled clinical trial in adult periodontitis patients. J. Periodontol. 2005, 76, 129-133. [CrossRef] [PubMed]

24. Guida, L.; Annunziata, M.; Belardo, S.; Farina, R.; Scabbia, A.; Trombelli, L. Effect of autogenous cortical bone particulate in conjunction with enamel matrix derivative in the treatment of periodontal intraosseous defects. J. Periodontol. 2007, 78, 231-238. [CrossRef] [PubMed]

25. Kim, C.K.; Chai, J.K.; Cho, K.S.; Moon, I.S.; Choi, S.H.; Sottosanti, J.S.; Wikesjo, U.M. Periodontal repair in intrabony defects treated with a calcium sulfate implant and calcium sulfate barrier. J. Periodontol. 1998, 69, 1317-1324. [CrossRef] [PubMed]

26. Zybutz, M.D.; Laurell, L.; Rapoport, D.A.; Persson, G.R. Treatment of intrabony defects with resorbable materials, non-resorbable materials and flap debridement. J. Clin. Periodontol. 2000, 27, 169-178. [CrossRef]

27. Tonetti, M.S.; Lang, N.P.; Cortellini, P.; Susan, J.E.; Adriaens, P.; Dubravec, D.; Fonzar, A.; Fourmousis, I.; Mayfield, L.; Rossi, R.; et al. Enamel matrix proteins in the regenerative therapy of deep intrabony defects. J. Clin. Periodontol. 2002, 29, 317-325. [CrossRef]

28. Mengel, R.; Soffner, M.; Flores-de-Jacoby, L. Bioabsorbable membrane and bioactive glass in the treatment of intrabony defects in patients with generalized aggressive periodontitis: Results of a 12-month clinical and radiological study. J. Periodontol. 2003, 74, 899-908. [CrossRef] 
29. Sanz, M.; Tonetti, M.S.; Zabalegui, I.; Sicilia, A.; Blanco, J.; Rebelo, H.; Rasperini, G.; Merli, M.; Cortellini, P.; Suvan, J.E. Treatment of Intrabony Defects with Enamel Matrix Proteins or Barrier Membranes: Results from a Multicenter Practice-Based Clinical Trial. J. Periodontol. 2004, 75, 726-733. [CrossRef]

30. Fickl, S.; Thalmair, T.; Kebschull, M.; Bohm, S.; Wachtei, H. Microsurgical access flap in conjunction with enamel matrix derivative for the treatment of intra-bony defects: A controlled clinical trial. J. Clin. Periodontol. 2009, 36, 784-790. [CrossRef]

31. Jepsen, S.; Topoll, H.; Rengers, H.; Heinz, B.; Teich, M.; Hoffmann, T.; Al-Machot, E.; Meyle, J.; Jervøe-Storm, P.M. Clinical outcomes after treatment of intra-bony defects with an EMD/synthetic bone graft or EMD alone: A multicentre randomizedcontrolled clinical trial. J. Clin. Periodontol. 2008, 35, 420-428. [CrossRef]

32. Meyle, J.; Hoffman, T.; Topoll, H.; Heinz, B.; Al-Machot, E.; Jevrøe-Storm, P.M.; Meiss, C.; Eickholz, P.; Jepsen, S. A multi-centre randomized controlled clinical trial on the treatment of intra-bony defects with enamel matrix derivatives/synthetic bone graft or enamel matrix derivatives alone: Results after 12 months. J. Clin. Periodontol. 2011, 38, 652-660. [CrossRef]

33. Christgau, M.; Schmalz, G.; Wenzel, A.; Hiller, K.A. Periodontal regeneration of intrabony defects with resorbable and nonresorbable membranes: 30-month results. J. Clin. Periodontol. 1997, 24, 17-27. [CrossRef]

34. Francetti, L.; Del Fabbro, M.; Basso, M.; Testori, T.; Weinstein, R. Enamel matrix pro-teins in the treatment of intra-bony defects. A prospective 24-month clinical trial. J. Clin. Periodontol. 2004, 31, 52-59. [CrossRef]

35. Siciliano, V.I.; Andreuccetti, G.; Siciliano, A.I.; Blasi, A.; Sculean, A.; Salvi, G.E. Clinical Outcomes After Treatment of NonContained Intrabony Defects with Enamel Matrix Derivative or Guided Tissue Regeneration: A 12-Month Randomized Controlled Clinical Trial. J. Periodontol. 2011, 82, 62-71. [CrossRef]

36. Bouchard, P.; Ouhayoun, J.P.; Nilvéus, R.E. Expanded polytet-rafluoroethylene membranes and connective tissue grafts support bone regeneration for closing mandibu-lar Class II furcations. J. Periodontol. 1993, 64, 1193-1198. [CrossRef]

37. Bouchard, P.; Giovannoli, J.-L.; Mattout, C.; Davarpanah, M.; Etienne, D. Clinical evaluation of a bioabsorbable regenerative material in mandibular class II furcation therapy. J. Clin. Periodontol. 1997, 24, 511-518. [CrossRef]

38. Garrett, S.; Polson, A.M.; Stoller, N.H.; Drisko, C.L.; Caton, J.G.; Harrold, C.Q.; Bogle, G.; Greenwell, H.; Lowenguth, R.A.; Duke, S.P.; et al. Comparison of a Bioabsorbable GTR Barrier to a Non-Absorbable Barrier in Treating Human Class II Furcation Defects. A Multi-Center Parallel Design Randomized Single-Blind Trial. J. Periodontol. 1997, 68, 667-675. [CrossRef]

39. Hugoson, A.; Ravald, N.; Fornell, J.; Johard, G.; Teiwik, A.; Gottlow, J. Treatment of Class II Furcation Involvements in Humans with Bioresorbable and Nonresorbable Guided Tissue Regeneration Barriers. A Randomized Multi-Center Study. J. Periodontol. 1995, 66, 624-634. [CrossRef]

40. Karapataki, S.; Falk, H.; Hugoso, A.; Olsson, G.; Slotte, C. Treatment of class II fur-cation defects using resorbable and nonresorbable GTR barriers. Swed. Dent. J. 1999, 23, 173-183.

41. Jepsen, S.; Heinz, B.; Jepsen, K.; Arjomand, M.; Hoffmann, T.; Richter, S.; Reich, E.; Sculean, A.; Gonzales, J.R.; Bödeker, R.H.; et al. A Randomized Clinical Trial Comparing Enamel Matrix Derivative and Membrane Treatment of Buccal Class II Furcation Involvement in Mandibular Molars. Part I: Study Design and Results for Primary Outcomes. J. Periodontol. 2004, 75, 1150-1160. [CrossRef]

42. Rams, T.E.; Degener, J.E.; Van Winkelhoff, A.J. Antibiotic Resistance in Human Chronic Periodontitis Microbiota. J. Periodontol. 2014, 85, 160-169. [CrossRef]

43. Ardila, C.-M.; Bedoya-García, J.-A. Antimicrobial resistance of Aggregatibacter actinomycetemcomitans, Porphyromonas gingivalis and Tannerella forsythia in periodontitis patients. J. Glob. Antimicrob. Resist. 2020, 22, 215-218. [CrossRef]

44. Jepsen, K.; Falk, W.; Brune, F.; Fimmers, R.; Jepsen, S.; Bekeredjian-Ding, I. Prevalence and antibiotic susceptibility trends of periodontal pathogens in the subgingival microbiota of German periodontitis patients: A retrospective surveillance study. J. Clin. Periodontol. 2021, 48, 1216-1227. [CrossRef]

45. Dyar, O.J.; Huttner, B.; Schouten, J.; Pulcini, C. What is antimicrobial stewardship? Clin. Microbiol. Infect. 2017, 23, 793-798. [CrossRef]

46. Bianco, A.; Cautela, V.; Napolitano, F.; Licata, F.; Pavia, M. Appropriateness of Antibiotic Prescription for Prophylactic Purposes among Italian Dental Practitioners: Results from a Cross-Sectional Study. Antibiotics 2021, 10, 547. [CrossRef]

47. Buonavoglia, A.; Leone, P.; Solimando, A.; Fasano, R.; Malerba, E.; Prete, M.; Corrente, M.; Prati, C.; Vacca, A.; Racanelli, V. Antibiotics or No Antibiotics, That Is the Question: An Update on Efficient and Effective Use of Antibiotics in Dental Practice. Antibiotics 2021, 10, 550. [CrossRef] 